\title{
Error representation of the time-marching DPG scheme
}

\author{
Judit Muñoz-Matute ${ }^{\mathrm{a}, \mathrm{b}}$, Leszek Demkowicz $^{\mathrm{b}}$, David Pardo ${ }^{\mathrm{c}, \mathrm{a}, \mathrm{d}}$ \\ ${ }^{a}$ Basque Center for Applied Mathematics (BCAM), Bilbao, Spain \\ ${ }^{b}$ Oden Institute for Computational Engineering and Sciences, \\ The University of Texas at Austin, Austin, USA \\ ${ }^{c}$ University of the Basque Country (UPV/EHU), Leioa, Spain \\ ${ }^{d}$ IKERBASQUE, Basque Foundation for Science, Bilbao, Spain
}

\begin{abstract}
In this article, we introduce an error representation function to perform adaptivity in time of the recently developed time-marching Discontinuous Petrov-Galerkin (DPG) scheme. We first provide an analytical expression for the error that is the Riesz representation of the residual. Then, we approximate the error by enriching the test space in such a way that it contains the optimal test functions. The local error contributions can be efficiently computed by adding a few equations to the time-marching scheme. We analyze the quality of such approximation by constructing a Fortin operator and providing an a posteriori error estimate. The time-marching scheme proposed in this article provides an optimal solution along with a set of efficient and reliable local error contributions to perform adaptivity. We validate our method for both parabolic and hyperbolic problems.
\end{abstract}

Keywords: DPG method, Error representation, Ultraweak formulation, Optimal test functions, Exponential integrators, Fortin operator

\section{Introduction}

The Discontinuous Petrov-Galerkin (DPG) method with optimal test functions is a well established method $[10,11]$ to approximate the solution of Partial Differential Equations (PDEs) proposed by Prof. Demkowicz and Gopalakrishnan about a decade ago [7, 9]. The principal idea is to construct optimal test functions in such a way that the discrete stability of the method is guaranteed. It has been applied in many frameworks [1, 4, 5, 8, 13, 16, 27]. It is well known that the DPG method can be interpreted as a minimum residual method and also as a mixed problem. In the latter, selecting an enriched test space, the method delivers a stable solution and a built-in error representation usually employed to perform adaptivity $[6,12,15,36]$.

There are previous articles about applying the DPG ideas to time-dependent problems. In $[14,18,19,26]$, the authors apply the DPG method in the space-time domain, enabling local space-time refinements. Conversely, authors in [22, 23, 37] apply and analyze the DPG method in space together with different time-stepping schemes for parabolic problems. In other works like $[24,38-40]$, the authors employ DPG-related ideas for solving both 
transient and frequency-domain problems, employing minimum residual methods or the corresponding mixed problems.

Recently, in [33, 34], we developed a time-marching scheme based on the DPG method for transient parabolic and hyperbolic problems, respectively. The main idea of the method is to apply the DPG technology only in the time variable to the system of Ordinary Differential Equations (ODEs) obtained after semidiscretizing in space a PDE with a BubnovGalerkin method. For that, we first consider a broken ultraweak variational formulation of the problem where, in the hyperbolic case, we reduce it first to a first order system. Then, the selection of the adjoint norm in the test space allows us to compute the optimal test functions analytically as we are considering a 1D problem. We found that the optimal test functions corresponding to piecewise polynomials for the trial space are exponentials of the stiffness matrix coming from the space discretization. We proved that the equation to compute the trace variables is equivalent to Exponential Integrators [29-31]. Moreover, the solution in the element interiors delivers an $L^{2}$-projection of the exact solution. In order to employ the existing software [28] available to compute exponential-related functions, we relate the optimal test functions to the so-called $\varphi$-functions employed in exponential time-integrators. Summarizing, we developed a time-marching scheme that is an exponential integrator for the traces and, additionally, we can compute the element interiors.

In this work, we present an error representation that we employ to perform adaptivity in time for the time-marching DPG scheme we introduced in [33, 34]. We know from the DPG community that the DPG solution minimizes the residual of the problem in the dual norm. It is well known that the built-in error representation function in DPG is the Riesz representation of the residual [10]. Here, as we are considering a 1D problem, we can also compute this error representation function analytically. We give an explicit expression of the error for any discrete solution in the trial space as well as for the optimal solution delivered by the DPG time-marching scheme. However, these expressions are given in integral form and their use requires a suitable study of appropriate quadrature rules.

Instead, we adopt the so-called practical $D P G[25]$ philosophy and we approximate the error representation function by enriching the test space. By doing so, we can compute the local error contributions as we solve the problem in the time-marching scheme by adding a few equations to the system. We construct a global Fortin operator, which is an orthogonal projection, and a-posteriori error estimation similar to [3] to prove that our approximation to the analytical error is reliable and efficient. We emphasize that we enrich the test space in such a way that it contains the analytical optimal test functions. Therefore, the timemarching scheme delivers the optimal solution and an approximation of the error. For that reason, we employ the Fortin constant for the a posteriori error estimation only and in this case, it does not affect the stability of the solution. We employ the local contributions of this approximated error to perform adaptivity in time via the Dörfler marking strategy [17]. We validate our adaptive method in both parabolic and hyperbolic problems.

This article is organized as follows: Section 2 presents the model problem and summarizes the time-marching scheme we developed in $[33,34]$. In Section 3 we show the analytical error representation function for any discrete ansatz solution on the discrete trial space and, 
in particular, for the optimal solution obtained with our time-marching scheme. Section 4 introduces a practical error representation function for the solution of the DPG method that we employ for adaptivity. Section 5 analyzes the approximation of the practical error to the analytical one by constructing a Fortin operator and developing an a posteriori error estimate. Section 6 presents numerical results of performing time adaptivity for both parabolic and hyperbolic problems. Finally, in Section 7, we summarize the conclusions and future work.

\section{Time-marching DPG scheme}

This section overviews the time-marching DPG scheme we introduced in [34] for parabolic problems. For simplicity, we consider a single Ordinary Differential Equation (ODE). The generalizations to hyperbolic problems and to systems of ODEs coming from the semidiscretization in space by the Bubnov-Galerkin method of Partial Differential Equations (PDEs) are summarized in [33, 34].

\subsection{Model problem and variational setting}

Let $I=(0,1] \subset \mathbb{R}$. We consider the following first order ODE

$$
\left\{\begin{aligned}
u^{\prime}+\lambda u & =f \quad \text { in } I, \\
u(0) & =u_{0},
\end{aligned}\right.
$$

where $\lambda \in \mathbb{R} \backslash\{0\}, u_{0} \in \mathbb{R}$ and $f \in L^{2}(I)$ are given data.

We define a partition $I_{h}$ of $I$ as

$$
0=t_{0}<t_{1}<\ldots<t_{m-1}<t_{m}=1,
$$

where $I_{k}=\left(t_{k-1}, t_{k}\right)$ and $h_{k}=t_{k}-t_{k-1}, \forall k=1, \ldots, m$. Related to this partition, we introduce the following trial and test (broken) spaces

$$
\begin{aligned}
& U=U^{0} \times \hat{U}=L^{2}(I) \times \mathbb{R}^{m}, \\
& V=H^{1}\left(I_{h}\right)=\left\{v \in L^{2}(I) \mid v_{\left.\right|_{I_{k}}} \in H^{1}\left(I_{k}\right), \forall I_{k} \in I_{h}\right\} .
\end{aligned}
$$

We define the jumps of a function $v \in V$ at each time $t_{k}$ as

$$
\begin{aligned}
& {[v]_{k}=v\left(t_{k}^{+}\right)-v\left(t_{k}^{-}\right), \quad \forall k=1, \ldots, m-1,} \\
& {[v]_{m}=-v\left(t_{m}^{-}\right),}
\end{aligned}
$$

where $v\left(t_{k}^{ \pm}\right):=\lim _{\varepsilon \rightarrow 0^{+}} v\left(t_{k} \pm \varepsilon\right)$. We consider an ultraweak variation formulation of (1) that reads

$$
\left\{\begin{array}{l}
\text { Find } z=\left(u, \hat{u}^{1}, \ldots, \hat{u}^{m}\right) \in U \text { such that } \\
b(z, v)=l(v), \quad \forall v \in V,
\end{array}\right.
$$


where

$$
\begin{aligned}
b(z, v) & :=\sum_{k=1}^{m} \int_{I_{k}} u\left(-v^{\prime}+\lambda v\right) d t-\hat{u}^{k}[v]_{k}, \\
l(v) & :=\sum_{k=1}^{m} \int_{I_{k}} f v d t+u_{0} v\left(0^{+}\right) .
\end{aligned}
$$

Finally, we consider the following trial and test norms

$$
\begin{aligned}
& \|z\|_{U}^{2}=\|u\|^{2}+\sum_{k=1}^{m}\left|\hat{u}^{k}\right|^{2} \\
& \|v\|_{V}^{2}=\sum_{k=1}^{m} \int_{I_{k}}\left|-v^{\prime}+\lambda v\right|^{2} d t+[v]_{k}^{2} .
\end{aligned}
$$

\subsection{Optimal test spaces and discrete scheme}

We select a discrete trial space $U_{h, p}=U_{h, p}^{0} \times \hat{U} \subset U$, where $U_{h, p}^{0}$ is composed of piecewise polynomials of order $p$. We define an element $z_{h}=\left(u_{h}, \hat{u}_{h}^{1}, \ldots, \hat{u}_{h}^{m}\right) \in U_{h, p}$ (see Figure 1) where

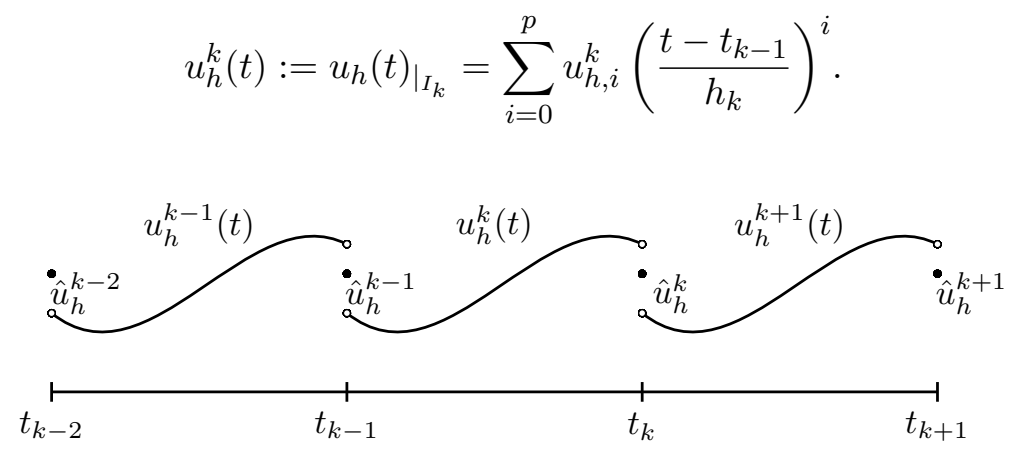

Figure 1: Approximated solution.

We define the optimal test space $V_{h, p}^{o p t} \subset V$ corresponding to the trial space $U_{h, p}$ by the span of the functions solving the following problem: Given $z_{h} \in U_{h, p}$

$$
\left\{\begin{array}{l}
\text { Find } v \in V \text { such that } \\
(v, \delta v)_{V}=b\left(z_{h}, \delta v\right), \quad \forall \delta v \in V,
\end{array}\right.
$$

where $(\cdot, \cdot)_{V}$ is the inner product corresponding to the adjoint test norm defined in $(6)$. We proved in [34] that

$$
V_{h, p}^{o p t}=\operatorname{span}\left\{\hat{v}^{k}, v_{j}^{k}, \forall j=0, \ldots, p, \forall k=1, \ldots, m\right\},
$$


where the optimal test functions can be defined recursively as

$$
\begin{aligned}
\hat{v}^{k}(t) & =e^{\lambda\left(t-t_{k}\right)}, \quad \forall t \in I_{k}, \\
v_{j}^{k}(t) & =\frac{1}{\lambda}\left(\left(\frac{t-t_{k-1}}{h_{k}}\right)^{j}+\frac{j}{h_{k}} v_{j-1}^{k}(t)-\hat{v}^{k}(t)\right), \quad \forall t \in I_{k} .
\end{aligned}
$$

Moreover, the optimal test functions satisfy the adjoint equation

$$
\left\{\begin{array}{c}
-\left(\hat{v}^{k}\right)^{\prime}+\lambda \hat{v}^{k}=0, \quad \hat{v}^{k}\left(t_{k}\right)=1, \\
-\left(v_{j}^{k}\right)^{\prime}+\lambda v_{j}^{k}=\left(\frac{t-t_{k-1}}{h_{k}}\right)^{j}, \quad v_{j}^{k}\left(t_{k}\right)=0, \forall j=0, \ldots, p .
\end{array}\right.
$$

Finally, we solve

$$
\left\{\begin{array}{l}
\text { Find } z_{h}=\left(u_{h}, \hat{u}_{h}^{1}, \ldots, \hat{u}_{h}^{m}\right) \in U_{h, p} \text { such that } \\
b\left(z_{h}, v_{h}\right)=l\left(v_{h}\right), \quad \forall v_{h} \in V_{h, p}^{\text {opt }}
\end{array}\right.
$$

and we obtain the following equivalent DPG-based time-marching scheme $\forall k=1, \ldots, m$

$$
\left\{\begin{array}{c}
\hat{u}_{h}^{k}=\hat{u}_{h}^{k-1} \hat{v}^{k}\left(t_{k-1}\right)+\int_{I_{k}} f \hat{v}^{k} d t, \\
\sum_{i=0}^{p} u_{h, i}^{k} \frac{h_{k}}{i+j+1}=\hat{u}_{h}^{k-1} v_{j}^{k}\left(t_{k-1}\right)+\int_{I_{k}} f v_{j}^{k} d t, \forall j=0, \ldots, p,
\end{array}\right.
$$

where $\hat{u}_{h}^{0}=u_{0}$.

The optimal test functions (8) for PDEs are exponentials of the stiffness matrix that we obtain after semidiscretizing in space by a Bubnov-Galerkin method. We proved in [33, 34] that the optimal test functions (8) are linear combinations of the so-called $\varphi$-functions employed in exponential integrators. In practice, we employ the software available from the exponential integrators community [2] to implement the DPG time-marching scheme (11).

\section{Analytical error representation}

It is well known in the DPG community [10] that the DPG method delivers an error representation function $\psi \in V$ whose norm equals the energy norm of the error of the solution, i.e.,

$$
\left\|z-z_{h}\right\|_{E}=\|\psi\|_{V}
$$

where the energy norm is defined as $\|z\|_{E}:=\sup _{0 \neq v \in V} \frac{|b(z, v)|}{\|v\|_{V}}$. The function $\psi$ is called the error representation function and it can be employed to perform adaptivity. It is defined as the solution of the following problem: Given $z_{h} \in U_{h, p}$

$$
\left\{\begin{array}{l}
\text { Find } \psi \in V \text { such that } \\
(\psi, \delta v)_{V}=b\left(z_{h}, \delta v\right)-l(\delta v), \quad \forall \delta v \in V .
\end{array}\right.
$$


Function $\psi$ is thus the Riesz representation of the residual. Definition (13) holds for any approximation $z_{h} \in U_{h, p}$, including the optimal solution given in (10). For simplicity in the notation, we employ $z_{h}$ in both cases specifying if it is the optimal solution of (10) or a perturbation of it. Similarly, $\psi$ denotes the analytical error representation function of both the solution of (10) or any perturbation of it, specifying each case accordingly. As in problem (7), we can solve (13) analytically.

Proposition 1. The error representation function (13) for any $z_{h} \in U_{h, p}$ with the variational setting defined in Section 2 is $\forall k=1, \ldots, m$

$$
\psi^{k}(t):=\psi(t)_{I_{k}}=C_{1}^{k} e^{\lambda t}+C_{2}^{k} e^{-\lambda t}+\frac{e^{\lambda t}}{2 \lambda} \int_{t}^{t_{k}} e^{-\lambda s} R^{k}(s) d s-\frac{e^{-\lambda t}}{2 \lambda} \int_{t}^{t_{k}} e^{\lambda s} R^{k}(s) d s,
$$

where $R^{k}(t):=\left(u_{h}^{k}(t)\right)^{\prime}+\lambda u_{h}^{k}(t)-f(t)_{\left.\right|_{I_{k}}}$ is the residual at $I_{k}$ and constants $C_{1}^{k}$ and $C_{2}^{k}$ are defined recursively as

$$
\begin{gathered}
\left\{\begin{array}{c}
C_{2}^{1}=I_{2}^{1}+\frac{e^{\lambda t_{0}}}{2 \lambda} S_{+}^{0}, \\
C_{2}^{k+1}=I_{2}^{k+1}+C_{2}^{k}+\frac{e^{\lambda t_{k}}}{2 \lambda}\left[u_{h}\right]_{k}, \quad \forall k=1, \ldots, m-1,
\end{array}\right. \\
\left\{\begin{array}{l}
C_{1}^{m}=(2 \lambda-1) C_{2}^{m} e^{-2 \lambda t_{m}}+e^{-\lambda t_{m}} S_{-}^{m}, \\
C_{1}^{k}=C_{1}^{k+1}+I_{1}^{k+1}+2 \lambda C_{2}^{k} e^{-2 \lambda t_{k}}+\frac{e^{-\lambda t_{k}}}{2 \lambda}\left[u_{h}\right]_{k}+e^{-\lambda t_{k}} S_{-}^{k}, \quad \forall k=m-1, \ldots, 1 .
\end{array}\right.
\end{gathered}
$$

Here, we denote $\forall k=1, \ldots, m$

$$
I_{1}^{k}=\frac{1}{2 \lambda} \int_{I_{k}} e^{-\lambda s} R^{k}(s) d s, \quad I_{2}^{k}=\frac{1}{2 \lambda} \int_{I_{k}} e^{\lambda s} R^{k}(s) d s,
$$

and also $S_{+}^{0}=u_{h}\left(t_{0}^{+}\right)-u_{0}$ and $\left[u_{h}\right]_{k}=S_{+}^{k}+S_{-}^{k}$ with

$$
S_{-}^{k}=\hat{u}_{h}^{k}-u_{h}\left(t_{k}^{-}\right), \quad S_{+}^{k}=u_{h}\left(t_{k}^{+}\right)-\hat{u}_{h}^{k} .
$$

Proof. Problem (13) reads

$$
\begin{aligned}
\sum_{k=1}^{m} \int_{I_{k}}\left(-\psi^{\prime}+\lambda \psi\right)\left(-\delta v^{\prime}+\lambda \delta v\right) d t+[\psi]_{k}[\delta v]_{k} & =\sum_{k=1}^{m} \int_{I_{k}} u_{h}\left(-\delta v^{\prime}+\lambda \delta v\right) d t-\hat{u}_{h}^{k}[\delta v]_{k} \\
& -\sum_{k=1}^{m} \int_{I_{k}} f \delta v d t-u_{0} \delta v\left(t_{0}^{+}\right),
\end{aligned}
$$

and selecting test functions with local support in $I_{k}$, we obtain

$$
\begin{aligned}
& \int_{I_{k}}\left(-\psi^{\prime}+\lambda \psi\right)\left(-\delta v^{\prime}+\lambda \delta v\right) d t-[\psi]_{k} \delta v\left(t_{k}^{-}\right)+[\psi]_{k-1} \delta v\left(t_{k-1}^{+}\right) \\
& =\int_{I_{k}} u_{h}\left(-\delta v^{\prime}+\lambda \delta v\right) d t+\hat{u}_{h}^{k} \delta v\left(t_{k}^{-}\right)-\hat{u}_{h}^{k-1} \delta v\left(t_{k-1}^{+}\right)-\int_{I_{k}} f \delta v d t .
\end{aligned}
$$


Integrating by parts, we have that $\psi(t)$ satisfies the following $m$ overlapping boundary value problems

$$
\left\{\begin{aligned}
-\psi^{\prime \prime}+\lambda^{2} \psi & =R^{k}, \quad \forall t \in I_{k}, \\
-\psi^{\prime}\left(t_{k-1}^{+}\right)+\lambda \psi\left(t_{k-1}^{+}\right)+\psi\left(t_{k-1}^{+}\right)-\psi\left(t_{k-1}^{-}\right) & =S_{+}^{k-1}, \quad\left(B C_{1}^{k}\right) \\
\psi^{\prime}\left(t_{k}^{-}\right)-\lambda \psi\left(t_{k}^{-}\right)+\psi\left(t_{k}^{-}\right)-\psi\left(t_{k}^{+}\right) & =S_{-}^{k}, \quad\left(B C_{2}^{k}\right)
\end{aligned}\right.
$$

$\forall k=1, \ldots, m$. In particular, for $k=1$ and $k=m$, we have

$$
\begin{aligned}
& B C_{1}^{1}:-\psi^{\prime}\left(t_{0}^{+}\right)+\lambda \psi\left(t_{0}^{+}\right)=S_{+}^{0}, \\
& B C_{2}^{m}: \psi^{\prime}\left(t_{m}^{-}\right)-\lambda \psi\left(t_{m}^{-}\right)+\psi\left(t_{m}^{-}\right)=S_{-}^{m} .
\end{aligned}
$$

From the first equation of (18), we obtain

$$
\psi^{k}(t)=C_{1}^{k} e^{\lambda t}+C_{2}^{k} e^{-\lambda t}+\frac{e^{\lambda t}}{2 \lambda} \int_{t}^{t_{k}} e^{-\lambda s} R^{k}(s) d s-\frac{e^{-\lambda t}}{2 \lambda} \int_{t}^{t_{k}} e^{\lambda s} R^{k}(s) d s,
$$

and we now determine $C_{1}^{k}$ and $C_{2}^{k}$ from the boundary conditions. From $B C_{1}^{k}$ in (18), we have $\forall k=2, \ldots, m$

$$
\left(C_{1}^{k}+I_{1}^{k}\right) e^{\lambda t_{k-1}}+\left(C_{2}^{k}-I_{2}^{k}\right)(1+2 \lambda) e^{-\lambda t_{k-1}}=C_{1}^{k-1} e^{\lambda t_{k-1}}+C_{2}^{k-1} e^{-\lambda t_{k-1}}+S_{+}^{k-1},
$$

and from $B C_{1}^{1}$ in (19)

$$
2 \lambda\left(C_{2}^{1}-I_{2}^{1}\right) e^{-\lambda t_{0}}=S_{+}^{0},
$$

which is the first equation in (15). Similarly, from $B C_{2}^{k}$ in (18) we obtain $\forall k=1, \ldots, m-1$

$$
C_{1}^{k} e^{\lambda t_{k}}+C_{2}^{k}(1-2 \lambda) e^{-\lambda t_{k}}-S_{-}^{k}=\left(C_{1}^{k+1}+I_{1}^{k+1}\right) e^{\lambda t_{k}}+\left(C_{2}^{k+1}-I_{2}^{k+1}\right) e^{-\lambda t_{k}}
$$

and from $B C_{2}^{m}$ in (19)

$$
C_{1}^{m} e^{\lambda t_{m}}+C_{2}^{m}(1-2 \lambda) e^{-\lambda t_{m}}=S_{-}^{m},
$$

which is the first equation in (16). From (20) and (21), we obtain the following system after adjusting the indices $\forall k=1, \ldots, m-1$

$$
\left\{\begin{array}{l}
\left(C_{1}^{k+1}+I_{1}^{k+1}\right) e^{\lambda t_{k}}+\left(C_{2}^{k+1}-I_{2}^{k+1}\right)(1+2 \lambda) e^{-\lambda t_{k}}=C_{1}^{k} e^{\lambda t_{k}}+C_{2}^{k} e^{-\lambda t_{k}}+S_{+}^{k}, \\
\left(C_{1}^{k+1}+I_{1}^{k+1}\right) e^{\lambda t_{k}}+\left(C_{2}^{k+1}-I_{2}^{k+1}\right) e^{-\lambda t_{k}}=C_{1}^{k} e^{\lambda t_{k}}+C_{2}^{k}(1-2 \lambda) e^{-\lambda t_{k}}-S_{-}^{k},
\end{array}\right.
$$

Finally, subtracting both equations in (22), we obtain the second equation in (15) and solving for $C_{1}^{k}$ in (22), we obtain the second equation in (16).

Corollary 1. From Proposition 1, it holds that

$$
\begin{aligned}
\|\psi\|_{V}^{2} & =\sum_{k=1}^{m} \int_{I_{k}}\left|-\psi^{\prime}+\lambda \psi\right|^{2} d t+[\psi]_{k}^{2} \\
& =\sum_{k=1}^{m} \int_{I_{k}}\left|2 \lambda C_{2}^{k} e^{-\lambda t}-e^{-\lambda t} \int_{t}^{t_{k}} e^{\lambda s} R^{k}(s) d s\right|^{2} d t+\left(-2 \lambda C_{2}^{k} e^{-\lambda t_{k}}-S_{-}^{k}\right)^{2} .
\end{aligned}
$$


The error representation function $\psi$ of Proposition 1 is given for any discrete ansatz function in $U_{h, p}$. In view of (12), we can employ the adjoint norm of the error described in Corollary 1 to perform adaptivity. However, we have not used in any place of the proof that $z_{h}$ is the optimal solution. In the next proposition we give the expression for the error representation function when $z_{h}$ is the solution of problem (10).

Proposition 2. The error representation function (13) for the solution $z_{h} \in U_{h, p}$ of problem (10) with the variational setting defined in Section 2 is $\forall k=1, \ldots, m$

$$
\psi^{k}(t):=\psi(t)_{\left.\right|_{k}}=C_{1}^{k} e^{\lambda t}-\frac{S_{-}^{k}}{2 \lambda} e^{\lambda\left(t_{k}-t\right)}+\frac{e^{\lambda t}}{2 \lambda} \int_{t}^{t_{k}} e^{-\lambda s} R^{k}(s) d s-\frac{e^{-\lambda t}}{2 \lambda} \int_{t}^{t_{k}} e^{\lambda s} R^{k}(s) d s,
$$

where $R^{k}(t):=\left(u_{h}^{k}(t)\right)^{\prime}+\lambda u_{h}^{k}(t)-f(t)_{\left.\right|_{I_{k}}}$ is the residual at $I_{k}$ and constants $C_{1}^{k}$ are defined recursively as

$$
\left\{\begin{array}{l}
C_{1}^{m}=-\frac{S_{-}^{m}}{2 \lambda} e^{-\lambda t_{m}}, \\
C_{1}^{k}=C_{1}^{k+1}+I_{1}^{k+1}+\frac{e^{-\lambda t_{k}}}{2 \lambda}\left[u_{h}\right]_{k}, \quad \forall k=m-1, \ldots, 1 .
\end{array}\right.
$$

Here, we denote $\forall k=1, \ldots, m$

$$
I_{1}^{k}=\frac{1}{2 \lambda} \int_{I_{k}} e^{-\lambda s} R^{k}(s) d s,
$$

and also $S_{+}^{0}=u_{h}\left(t_{0}^{+}\right)-u_{0}$ and $\left[u_{h}\right]_{k}=S_{+}^{k}+S_{-}^{k}$ with

$$
S_{-}^{k}=\hat{u}_{h}^{k}-u_{h}\left(t_{k}^{-}\right), \quad S_{+}^{k}=u_{h}\left(t_{k}^{+}\right)-\hat{u}_{h}^{k} .
$$

Proof. From Proposition 1, we know that the error representation function $\psi(t)$ corresponding to the optimal solution $z_{h}$ of problem (10) is (14). Aditionally, as $z_{h}$ satisfies (10), we have that

$$
\left(\psi, \delta v_{h}\right)_{V}=0, \forall \delta v_{h} \in V_{h, p}^{o p t}
$$

In particular, testing with $\hat{v}^{k}$ in (17) and employing (9), we obtain

$$
\begin{aligned}
& {[\psi]_{1}=0,} \\
& {[\psi]_{k}=[\psi]_{k-1} \hat{v}^{k}\left(t_{k-1}\right), \quad \forall k=2, \ldots, m,}
\end{aligned}
$$

which implies that $[\psi]_{k}=0, \forall k=1, \ldots, m$ and, therefore, in this case $\psi(t)$ is a globally continuous function and from (23),

$$
[\psi]_{k}=-2 \lambda C_{2}^{k} e^{-\lambda t_{k}}-S_{-}^{k}=0, \forall k=1, \ldots, m,
$$

which lead to expressions (24) and (25). Finally, recurrence formulas (15) reduce to

$$
\frac{-e^{t_{k}}}{2 \lambda} S_{-}^{k}=I_{2}^{k}+\frac{e^{\lambda t_{k-1}}}{2 \lambda} S_{+}^{k-1}, \forall k=1, \ldots, m,
$$


and integrating by parts in $I_{2}^{k}$ we obtain

$$
2 \lambda I_{2}^{k}=e^{\lambda t_{k}} u_{h}\left(t_{k}^{-}\right)-e^{\lambda t_{k-1}} u_{h}\left(t_{k-1}^{+}\right)-\int_{I_{k}} f e^{\lambda t} d t
$$

and from the first equation in (11) we have that $\int_{I_{k}} f e^{\lambda t} d t=e^{\lambda t_{k}} \hat{u}_{h}^{k}-e^{\lambda t_{k-1}} \hat{u}_{h}^{k-1}$, therefore conditions (27) are automatically satisfied.

Corollary 2. From Proposition 2, it holds that

$$
\begin{aligned}
\|\psi\|_{V}^{2} & =\sum_{k=1}^{m} \int_{I_{k}}\left|-\psi^{\prime}+\lambda \psi\right|^{2} d t+[\psi]_{k}^{2} \\
& =\sum_{k=1}^{m} \int_{I_{k}}\left|-S_{-}^{k} e^{\lambda\left(t_{k}-t\right)}-e^{-\lambda t} \int_{t}^{t_{k}} e^{\lambda s} R^{k}(s) d s\right|^{2} d t .
\end{aligned}
$$

In this particular case, the error representation function (24) can be computed backwards in time and the adjoint norm (29) is a sum of local contributions. However, both expressions are given in integral form. In the next section, we introduce an approximation to the analytical error representation function with the goal of simplifying both $\psi(t)$ in $(24)$ and its adjoint norm (29).

\section{Practical error representation}

In order to obtain a computable approximation of the analytical error representation defined in Proposition 2, we consider the practical DPG framework [25] in this section. The idea is to select a finite dimensional subspace of $V$ to solve both problems (7) and (13). However, we can take advantage of knowing the analytical solution of both problems. Our goal is to find a subspace of $V$ that delivers the same optimal test functions as in (7) and a good approximation for (13).

Given the trial space $U_{h, p}$ defined in Section 2, we propose to solve both problems (7) and (13) substituting $V$ by the following subspace

$$
V_{h, r}=\operatorname{span}\left\{\hat{v}^{k}, v_{j}^{k}, \forall j=0, \ldots, r, \forall k=1, \ldots, m\right\},
$$

with $r \geq p+1$ and the functions in (30) satisfying (8) and (9). We now define the discrete version of (7) as

$$
\left\{\begin{array}{l}
\text { Find } v_{h} \in V_{h, r} \text { such that } \\
\left(v_{h}, \delta v_{h}\right)_{V}=b\left(z_{h}, \delta v_{h}\right), \quad \forall \delta v_{h} \in V_{h, r},
\end{array}\right.
$$

and the discrete version of (13) as

$$
\left\{\begin{array}{l}
\text { Find } \psi_{h} \in V_{h, r} \text { such that } \\
\left(\psi_{h}, \delta v_{h}\right)_{V}=b\left(z_{h}, \delta v_{h}\right)-l\left(\delta v_{h}\right), \quad \forall \delta v_{h} \in V_{h, r} .
\end{array}\right.
$$

This construction leads us to the following remarks, which are the key points of this article. 
Remark 1. The space defined in (30) is an enriched test space containing the optimal test functions corresponding to $U_{h, p}$, i.e. $V_{h, p}^{\text {opt }} \subset V_{h, r}$. It is easy to verify that the constants corresponding to $v_{j}^{k}$ with $j=p+1, \ldots, r$ are equal to zero in system (31). Therefore, the optimal test space we obtain from (31) is exactly $V_{h, p}^{\text {opt }}$ and we can conclude that practical $D P G$ method we propose here delivers the optimal solution (10).

Remark 2. We know from Propositions 1 and 2 that $\psi(t)$ includes negative exponential terms like $e^{-\lambda t}$, which is not an element of $V_{h, r}$. Therefore, problem (32) does not deliver $\psi(t) \in V$ but an approximation of it: $\psi_{h}(t) \in V_{h, r} \subset V$. We study this approximation in the next section by introducing a Fortin operator and a posteriori error estimates.

Summarizing, the practical DPG method defined in this section delivers the optimal solution and an approximation of the error representation function. Finally, we give an explicit formula to compute the approximated error $\psi_{h} \in V_{h, r}$ for the optimal solution in (10).

Proposition 3. The approximate error representation function (32) for the solution $z_{h} \in$ $U_{h, p}$ of problem (10) with the variational setting defined in Section 2 is

$$
\psi_{h}(t)=\sum_{k=1}^{m}\left(\hat{\psi}_{h}^{k} \hat{v}^{k}+\sum_{i=0}^{r} \psi_{h, i}^{k} v_{i}^{k}\right),
$$

with the coefficients satisfying the following local systems $\forall k=1, \ldots, m$

$$
\left\{\begin{array}{l}
\sum_{i=0}^{r} \frac{h_{k}}{i+j+1} \psi_{h, i}^{k}=0, \forall j=0, \ldots, p, \\
\sum_{i=0}^{r} \frac{h_{k}}{i+j+1} \psi_{h, i}^{k}=\sum_{i=0}^{p} \frac{h_{k}}{i+j+1} u_{h, i}^{k}-\int_{I_{k}} f^{k} v_{j}^{k} d t-\hat{u}_{h}^{k-1} v_{j}^{k}\left(t_{k-1}\right), \quad \forall j=p+1, \ldots, r,
\end{array}\right.
$$

and global continuity conditions

$$
\left\{\begin{array}{l}
\hat{\psi}_{h}^{m}=0 \\
\hat{\psi}_{h}^{k}=\hat{\psi}_{h}^{k+1} \hat{v}^{k+1}\left(t_{k}\right)+\sum_{i=0}^{r} \psi_{h, i}^{k+1} v_{i}^{k+1}\left(t_{k}\right), \quad \forall k=m-1, \ldots, 1 .
\end{array}\right.
$$

Proof. From conditions (9) of the basis functions in $V_{h, r}$, we have that

$$
-\psi_{h}^{\prime}+\lambda \psi_{h}=\sum_{k=1}^{m} \sum_{i=0}^{r} \psi_{h, i}^{k}\left(\frac{t-t_{k-1}}{h_{k}}\right)^{i},
$$

and also

$$
\left[\psi_{h}\right]_{k}=\psi_{h}\left(t_{k}^{+}\right)-\psi_{h}\left(t_{k}^{-}\right)=\hat{\psi}_{h}^{k+1} \hat{v}^{k+1}\left(t_{k}\right)+\sum_{i=0}^{r} \psi_{h, i}^{k+1} v_{i}^{k+1}\left(t_{k}\right)-\hat{\psi}_{h}^{k} .
$$


We note that the right-hand-side of (32) vanishes for all functions in $V_{h, p}^{\text {opt }}$, i.e.,

$$
\left(\psi_{h}, \delta v_{h}\right)_{V}=0, \quad \forall \delta v_{h} \in V_{h, p}^{o p t} \subset V_{h, r} .
$$

This follows directly from the fact that $z_{h}$ is the solution from problem (10).

We now test problem (32) with the basis functions of $V_{h, r}$ :

- If we test with $\hat{v}^{k}, \forall k=2, \ldots, m$, as $\hat{v}^{k} \in V_{h, p}^{o p t}$ and it has local support in $I_{k}$, we obtain

$$
\int_{I_{k}}\left(-\psi_{h}^{\prime}+\lambda \psi_{h}\right)\left(-\left(\hat{v}^{k}\right)^{\prime}+\lambda \hat{v}^{k}\right) d t-\left[\psi_{h}\right]_{k} \hat{v}^{k}\left(t_{k}\right)+\left[\psi_{h}\right]_{k-1} \hat{v}^{k}\left(t_{k-1}\right)=0
$$

and from (9), we have

$$
\left[\psi_{h}\right]_{k}=\left[\psi_{h}\right]_{k-1} \hat{v}^{k}\left(t_{k-1}\right), \quad \forall k=2, \ldots, m .
$$

In particular, for $\hat{v}^{1}$, as we do not have a jump in $t_{0}$, we obtain that $\left[\psi_{h}\right]_{1}=0$. Therefore, the jumps of $\psi_{h}$ vanish, i.e,

$$
\begin{aligned}
& {\left[\psi_{h}\right]_{k}=0, \quad \forall k=1, \ldots, m-1,} \\
& {\left[\psi_{h}\right]_{m}=-\hat{\psi}_{h}^{m}=0,}
\end{aligned}
$$

which are the conditions in (35) with the jumps defined in (37). We then conclude from $(35)$ that $\psi_{h}(t)$ is a globally continuous function.

- If we test with $v_{j}^{k}, \forall k=1, \ldots, m, \forall j=0, \ldots, p$, we have

$$
\int_{I_{k}}\left(-\psi_{h}^{\prime}+\lambda \psi_{h}\right)\left(-\left(\hat{v}^{k}\right)^{\prime}+\lambda \hat{v}^{k}\right) d t=0
$$

and from (36) and (9) we obtain the first equation in (34)

$$
\sum_{i=0}^{r} \psi_{h, i}^{k} \int_{I_{k}}\left(\frac{t-t_{k-1}}{h_{k}}\right)^{i+j} d t=0 .
$$

- Similarly, when we test with $v_{j}^{k}, \forall k=1, \ldots, m, \forall j=p+1, \ldots, r$, we obtain the second equation in (34)

$$
\sum_{i=0}^{r} \psi_{h, i}^{k} \int_{I_{k}}\left(\frac{t-t_{k-1}}{h_{k}}\right)^{i+j} d t=\sum_{i=0}^{p} u_{h, i}^{k} \int_{I_{k}}\left(\frac{t-t_{k-1}}{h_{k}}\right)^{i+j} d t-\int_{I_{k}} f^{k} v_{j}^{k} d t-\hat{u}_{h}^{k-1} v_{j}^{k}\left(t_{k-1}\right) .
$$


Corollary 3. From Proposition 3, it holds that

$$
\left\|\psi_{h}\right\|_{V}^{2}=\sum_{k=1}^{m} \int_{I_{k}}\left|-\psi_{h}^{\prime}+\lambda \psi_{h}\right|^{2} d t+\left[\psi_{h}\right]_{k}^{2}=\sum_{k=1}^{m} \int_{I_{k}}\left|\sum_{i=0}^{r} \psi_{h, i}^{k}\left(\frac{t-t_{k-1}}{h_{k}}\right)^{i}\right|^{2} d t .
$$

We observe that computing the error $\psi_{h}(t)$ in (33) involves another time-marching scheme that needs to be computed backwards in time. We know that $\hat{\psi}_{h}^{m}=0$, then we can compute the local problem (34) for $k=m$ to calculate $\psi_{h, i}^{m}$ and then employ (35) to compute $\hat{\psi}_{h}^{m-1}$. We repeat this process for $k=m, \ldots, 1$.

The error representation function $\psi_{h}(t)$ is globally continuous. However, $-\psi_{h}^{\prime}(t)+\lambda \psi_{h}(t)$ is a discontinuous function with piecewise polynomials of order $r$ in each element - see Eq. (9). Therefore, computing the adjoint norm of $\psi_{h}(t)$ in (39) reduces to solving local problems (34) and then integrate a polynomial of order $r$ in each element, which is simpler than the expression given in Proposition 2.

Remark 3. We can also employ (32) to approximate the error representation function of any perturbed solution $z_{h} \in U_{h, p}$. However, as it occurs in Proposition 1, the jumps are not zero and (32) is not a time-marching scheme but a global problem.

\section{Error analysis}

In this section, we analyze the approximation of the analytical error representation given in (32) by constructing a Fortin operator [35], and introducing a posteriori error estimates similar to [3].

\subsection{Fortin operator}

We first recall the notion of Fortin operator [21].

Definition 1. A linear map $\Pi: V \longrightarrow V_{h, r}$ is called a Fortin operator if it satisfies the following conditions

$$
\left\{\begin{array}{l}
b\left(\delta z_{h}, v-\Pi v\right)=0, \quad \forall \delta z_{h} \in U_{h, p}, \\
\|\Pi v\|_{V} \leq C_{\Pi}\|v\|_{V} .
\end{array}\right.
$$

The constant $C_{\Pi}$ is the operator norm and it is referred to as the Fortin constant.

In the next theorem, we construct a global Fortin operator defined in Definition 1 and we prove that it is an orthogonal projection, hence its norm is equal one.

Theorem 1. The following operator $\Pi: V \longrightarrow V_{h, r}$ defined locally at each element $I_{k}$ as

$$
\left\{\begin{aligned}
\int_{I_{m}} \chi_{h}\left(-\Pi v^{\prime}+\lambda \Pi v\right) d t & =\int_{I_{m}} \chi_{h}\left(-v^{\prime}+\lambda v\right) d t, \quad \forall \chi_{h} \in \mathcal{P}_{r}\left(I_{m}\right), \\
\Pi v\left(t_{m}^{-}\right) & =v\left(t_{m}^{-}\right),
\end{aligned}\right.
$$


and $\forall k=m-1, \ldots, 1$

$$
\left\{\begin{aligned}
\int_{I_{k}} \chi_{h}\left(-\Pi v^{\prime}+\lambda \Pi v\right) d t & =\int_{I_{k}} \chi_{h}\left(-v^{\prime}+\lambda v\right) d t, \quad \forall \chi_{h} \in \mathcal{P}_{r}\left(I_{k}\right), \\
\Pi v\left(t_{k}^{-}\right) & =\Pi v\left(t_{k}^{+}\right)-[v]_{k},
\end{aligned}\right.
$$

with $\mathcal{P}_{r}\left(I_{k}\right)$ denoting the space of polynomials up to order $r$ in $I_{k}$, satisfies conditions (40). Moreover, $\Pi$ is an orthogonal projection of functions from $V$ into $V_{h, r}$ and therefore

$$
C_{\Pi}=1 .
$$

Proof. Employing the test functions defined in (30), we express $\Pi v \in V_{h, r}$ as

$$
\Pi v=\sum_{k=1}^{m}\left(\hat{\alpha}^{k} \hat{v}^{k}+\sum_{i=0}^{r} \alpha_{i}^{k} v_{i}^{k}\right) .
$$

Therefore, (41) and (42) describe a square system of $m(r+2)$ unknowns and equations. We can solve (41) and (42) as a time-marching-scheme backwards in time and the value $\Pi v\left(t_{k}^{+}\right)$ is known from solving the system at $I_{k+1}$.

If we multiply the equations at the boundaries by real numbers $\hat{\chi}_{h}^{k} \in \mathbb{R}$ and summing up all equations in (41) and (42), we obtain

$\sum_{k=1}^{m} \int_{I_{k}} \chi_{h}\left(-\Pi v^{\prime}+\lambda \Pi v\right) d t-\hat{\chi}_{h}^{k}[\Pi v]_{k}=\sum_{k=1}^{m} \int_{I_{k}} \chi_{h}\left(-v^{\prime}+\lambda v\right) d t-\hat{\chi}_{h}^{k}[v]_{k}, \forall\left(\chi_{h}, \hat{\chi}_{h}^{1}, \ldots, \hat{\chi}_{h}^{m}\right) \in U_{h, r}$

or equivalently, $b\left(\delta \chi_{h}, \Pi v\right)=b\left(\delta \chi_{h}, v\right), \quad \forall \delta \chi_{h} \in U_{h, r}$. As $U_{h, p} \subset U_{h, r}$, the first condition in Definition 1 is satisfied.

From (9) and (43), we can rewrite (41) and (42) as

$$
\left\{\begin{aligned}
\sum_{i=0}^{r} \alpha_{i}^{m} \int_{I_{m}}\left(\frac{t-t_{m-1}}{h_{m}}\right)^{i+j} d t & =\int_{I_{m}}\left(\frac{t-t_{m-1}}{h_{m}}\right)^{j}\left(-v^{\prime}+\lambda v\right) d t, \forall j=0, \ldots, r, \\
\hat{\alpha}^{m} & =v\left(t_{m}^{-}\right),
\end{aligned}\right.
$$

and $\forall k=m-1, \ldots, 1$

$$
\left\{\begin{aligned}
\sum_{i=0}^{r} \alpha_{i}^{k} \int_{I_{k}}\left(\frac{t-t_{k-1}}{h_{k}}\right)^{i+j} d t & =\int_{I_{k}}\left(\frac{t-t_{k-1}}{h_{k}}\right)^{j}\left(-v^{\prime}+\lambda v\right) d t, \forall j=0, \ldots, r, \\
\hat{\alpha}^{k} & =\hat{\alpha}^{k+1} \hat{v}^{k+1}\left(t_{k}^{+}\right)+\sum_{i=0}^{r} \alpha^{k+1} v_{i}^{k+1}\left(t_{k}^{+}\right)-[v]_{k} .
\end{aligned}\right.
$$

To see that $\Pi$ is a projection, we select in the right-and-side of (44) and (45), $v=\Pi w \in V_{h, r}$ with $w \in V$ and we express

$$
\Pi w=\sum_{k=1}^{m}\left(\hat{\beta}^{k} \hat{v}^{k}+\sum_{i=0}^{r} \beta_{i}^{k} v_{i}^{k}\right)
$$


From (44) and the first equation in (45), it is easy to see that $\beta_{i}^{k}=\alpha_{i}^{k}, \forall k=1, \ldots, m, \forall i=$ $0, \ldots, r$, and $\hat{\beta}^{m}=\hat{\alpha}^{m}$. From the second equation in (45), we have

$$
\hat{\beta}^{k}=\hat{\beta}^{k+1} \hat{v}^{k+1}\left(t_{k}^{+}\right)+\sum_{i=0}^{r} \beta_{i}^{k+1} v_{i}^{k+1}\left(t_{k}^{+}\right)+\hat{\alpha}^{k}-\hat{\alpha}^{k+1} v\left(t_{k}^{+}\right)-\sum_{i=0}^{r} \alpha_{i}^{k+1} v_{i}^{k+1}\left(t_{k}^{+}\right),
$$

which reduces to $\hat{\beta}^{k}-\hat{\beta}^{k+1} \hat{v}^{k+1}\left(t_{k}^{+}\right)=\hat{\alpha}^{k}-\hat{\alpha}^{k+1} v^{k+1}\left(t_{k}^{+}\right), \forall k=m-1, \ldots, 1$. As $\hat{\beta}^{m}=\hat{\alpha}^{m}$, we have that $\hat{\beta}^{k}=\hat{\alpha}^{k}, \forall k=m-1, \ldots, 1$. Therefore, $\Pi(\Pi w)=\Pi w, \forall w \in V$ so $\Pi$ is a projection.

Finally, to prove that $\Pi$ is an orthogonal projection, we need to see that $(v-\Pi v, \Pi v)_{V}=$ $0, \forall v \in V$. Considering the inner product defined in (6), we have from (9) that the optimal test functions satisfy $\forall k=2, \ldots, m$

$$
\begin{aligned}
\left(\hat{v}^{k}, \delta v\right)_{V} & =-[\delta v]_{k}+\hat{v}^{k}\left(t_{k-1}^{+}\right)[\delta v]_{k-1}=b((0,0, \ldots \underbrace{1}_{k}, \ldots, 0), \delta v)+\hat{v}^{k}\left(t_{k-1}^{+}\right)[\delta v]_{k-1}, \\
\left(v_{i}^{k}, \delta v\right)_{V} & =\int_{I_{k}}\left(\frac{t-t_{k-1}}{h_{k}}\right)^{i}(-\delta v+\lambda \delta v) d t+v_{i}^{k}\left(t_{k-1}^{+}\right)[\delta v]_{k-1} \\
& =b\left(\left(\left(\frac{t-t_{k-1}}{h_{k}}\right)^{i}, 0, \ldots, 0\right), \delta v\right)+v_{i}^{k}\left(t_{k-1}^{+}\right)[\delta v]_{k-1}, \forall i=0, \ldots, r,
\end{aligned}
$$

and for $k=1$ we obtain the same expression but without the jump term. Therefore, from these equalities and the definition of $\Pi$ we obtain

$$
\begin{aligned}
(\Pi v, \Pi v)_{V} & =\sum_{k=1}^{m} \hat{\alpha}^{k}\left(\hat{v}^{k}, \Pi v\right)_{V}+\sum_{k=1}^{m} \sum_{i=0}^{r} \alpha_{i}^{k}\left(v_{i}^{k}, \Pi v\right)_{V} \\
& =\sum_{k=1}^{m} \hat{\alpha}^{k} b((0,0, \ldots, \underbrace{1}_{k}, \ldots, 0), \Pi v)+\sum_{k=2}^{m} \hat{v}^{k}\left(t_{k-1}^{+}\right)[\Pi v]_{k-1} \\
& +\sum_{k=1}^{m} \sum_{i=0}^{r} \alpha_{i}^{k} b\left(\left(\left(\frac{t-t_{k-1}}{h_{k}}\right)^{i}, 0, \ldots, 0\right), \Pi v\right)+\sum_{k=2}^{m} \sum_{i=0}^{r} v_{i}^{k}\left(t_{k-1}^{+}\right)[\Pi v]_{k-1} \\
& =\sum_{k=1}^{m} \hat{\alpha}^{k} b((0,0, \ldots, \underbrace{1}_{k}, \ldots, 0), v)+\sum_{k=2}^{m} \hat{v}^{k}\left(t_{k-1}^{+}\right)[v]_{k-1} \\
& +\sum_{k=1}^{m} \sum_{i=0}^{r} \alpha_{i}^{k} b\left(\left(\left(\frac{t-t_{k-1}}{h_{k}}\right)^{i}, 0, \ldots, 0\right), v\right)+\sum_{k=2}^{m} \sum_{i=0}^{r} v_{i}^{k}\left(t_{k-1}^{+}\right)[v]_{k-1} \\
& =\sum_{k=1}^{m} \hat{\alpha}^{k}\left(\hat{v}^{k}, v\right)_{V}+\sum_{k=1}^{m} \sum_{i=0}^{r} \alpha_{i}^{k}\left(v_{i}^{k}, v\right)_{V}=(\Pi v, v)_{V} .
\end{aligned}
$$




\subsection{A posteriori error estimation}

We first analyze the continuity and the inf-sup constants of the continuous broken formulation (4) in the next theorem.

Theorem 2. The bilinear form defined in (5) satisfies the following inf-sup and continuity conditions

$$
\gamma|| z\left\|_{U} \leq \sup _{0 \neq v \in V} \frac{|b(z, v)|}{\|v\|_{V}} \leq M\right\| z \|_{U}
$$

with $\gamma=M=1$ and the following uniqueness condition holds

$$
\{v \in V \mid b(z, v)=0, \forall z \in U\}=\{0\} .
$$

Proof. We first proof the uniqueness condition (47). We have that

$$
b(z, v)=\sum_{k=1}^{m} \int_{I_{k}} u\left(-v^{\prime}+\lambda v\right) d t-\hat{u}^{k}[v]_{k}=0, \quad \forall z \in U .
$$

In particular, it holds for $z=(0,1, \ldots, 1)$, therefore $[v]_{k}=0, \forall k=1, \ldots, m$. This means that $v \in H^{1}(\Omega)$ and $v\left(t_{m}\right)=0$. Now, selecting functions $u$ with local support in $I_{k}$, we obtain that $v(t)$ satisfies

$$
\left\{\begin{aligned}
-v^{\prime}+\lambda v & =0, \forall t \in I_{k}, \\
{[v]_{k} } & =0, \forall k=1, \ldots, m-1, \\
v\left(t_{m}\right) & =0
\end{aligned}\right.
$$

The first equation in (48) leads to $v(t)_{\left.\right|_{I_{k}}}=\alpha_{k} e^{\lambda\left(t-t_{k}\right)}, \forall k=1, \ldots, m$, and from the second equation in (48), we have

$$
\alpha_{k+1} e^{\lambda h_{k}}=\alpha_{k}, \quad \forall k=1, \ldots, m-1 .
$$

The last condition in (48) implies that $\alpha_{m}=0$ and therefore $\alpha_{k}=0, \forall k=1, \ldots, m$ and (47) holds. The continuity constant of the bilinear form holds directly from the CauchySchwarz inequality

$$
\begin{aligned}
\sup _{0 \neq v \in V} \frac{|b(z, v)|^{2}}{\|v\|_{V}^{2}} & =\sup _{0 \neq v \in V} \frac{\left|\sum_{k=1}^{m} \int_{I_{k}} u\left(-v^{\prime}+\lambda v\right) d t-\hat{u}^{k}[v]_{k}\right|^{2}}{\|v\|_{V}^{2}} \\
& \leq \sup _{0 \neq v \in V} \frac{\left(\sum_{k=1}^{m} \int_{I_{k}}|u|^{2} d t+\left|\hat{u}^{k}\right|^{2}\right)\left(\sum_{k=1}^{m} \int_{I_{k}}\left|-v^{\prime}+\lambda v\right|^{2} d t+[v]_{k}^{2}\right)}{\|v\|_{V}^{2}} \leq\|z\|_{U}^{2} .
\end{aligned}
$$


For the inf-sup condition, we test with $v=u^{\prime}+\lambda u$ in $I_{k}$ and $[v]_{k}=\hat{u}^{k}$ and we obtain

$$
\sup _{\substack{0 \neq v \in V \\ 0}} \frac{|b(z, v)|^{2}}{\|v\|_{V}^{2}} \geq \frac{\left.\left|\sum_{k=1}^{m} \int_{I_{k}}\right| u\right|^{2} d t+\left.\left|\hat{u}^{k}\right|^{2}\right|^{2}}{\sum_{k=1}^{m} \int_{I_{k}}|u|^{2} d t+\left|\hat{u}^{k}\right|^{2}}=\|z\|_{U}^{2} .
$$

In general, Theorem 2 implies that the analytical error representation function $\psi(t)$ provides a reliable and efficient error control of the error of the solution in the norm of $U$. In other words, $\|\psi\|_{V}$ is both an upper bound and a lower bound of $\left\|z-z_{h}\right\|_{U}$. It follows directly form (12) and (46)

$$
\frac{1}{M}\|\psi\|_{V} \leq\left\|z-z_{h}\right\|_{U} \leq \frac{1}{\gamma}\|\psi\|_{V}
$$

In this case, as $M=\gamma=1$, the equality $\|\psi\|_{V}=\left\|z-z_{h}\right\|_{U}$ holds.

The next theorem proves, following the arguments of the more general proofs in $[3,32]$, that the approximated error representation function $\psi_{h}(t)$ introduced in (32) is reliable and efficient. For that, we need the Fortin operator defined in Section 5.1.

Theorem 3. The approximated error representation function (32) provides a reliable and efficient error control of the analytical error representation function (13), i.e.,

$$
\left\|\psi_{h}\right\|_{V} \leq\|\psi\|_{V} \leq\left\|\psi_{h}\right\|_{V}+\text { osc. }
$$

Here, osc $=\|l \circ(I-\Pi)\|_{V^{\prime}}$ is the oscillation term where $V^{\prime}$ is the dual space of $V$ and $l \in V^{\prime}$ is the linear form defined in (5).

Proof. We first denote $\varepsilon=\psi-\psi_{h} \in V$. Restricting (13) to $V_{h, r}$ and subtracting (32), we obtain

$$
\left(\varepsilon, \delta v_{h}\right)_{V}=0, \quad \forall v_{h} \in V_{h, r} .
$$

In particular, $(\varepsilon, \Pi \psi)_{V}=0$, which implies that $\Pi \psi=\psi_{h}$ as $\Pi$ is an orthogonal projection. Therefore, $\Pi \varepsilon=0$ and the first inequality of (50) holds directly from (40). From (51), we have

$$
\|\psi\|_{V}^{2}=\|\varepsilon\|_{V}^{2}+\left\|\psi_{h}\right\|_{V}^{2}
$$

and also

$$
\begin{aligned}
\|\varepsilon\|_{V}^{2} & =(\varepsilon, \varepsilon)_{V}=\left(\psi-\psi_{h}, \varepsilon\right)_{V}=(\psi, \varepsilon)_{V}=(\psi, \varepsilon-\Pi \varepsilon)_{V} \\
& =l(\varepsilon-\Pi \varepsilon)-b\left(z_{h}, \varepsilon-\Pi \varepsilon\right)=l(\varepsilon-\Pi \varepsilon) .
\end{aligned}
$$

Therefore,

$$
\|\varepsilon\|_{V} \leq\|l \circ(I-\Pi)\|_{V^{\prime}}=o s c,
$$

and finally from (52), we obtain the second inequality in (50). 
Remark 4. In Theorem 3, the oscillation term osc measures the data approximation error. From [3, 32], we have the following bound

$$
o s c \leq M C_{\Pi} \min _{z_{h} \in U_{h, p}}\left\|z-z_{h}\right\|_{U}=\min _{z_{h} \in U_{h, p}}\left\|z-z_{h}\right\|_{U} .
$$

Numerical results show that an enrichment in the order of the test space improves the approximation of the practical error to the analytical one, which implies that the oscillation term becomes negligible. For practical purposes, we set $r=p+1$ in our experiments.

Remark 5. The construction of the Fortin operator in this section is consistent with the fact that our practical DPG method delivers the optimal solution. We know from [10] that the existence of a Fortin operator implies the discrete inf-sup condition with $\gamma_{h} \geq \frac{\gamma}{C_{\Pi}}$. In our case, as we proved that $C_{\Pi}=1$, we have that

$$
\gamma_{h} \geq \frac{\gamma}{C_{\Pi}}=\gamma
$$

Conversely, as the optimal test space for $U_{h, p}$ is contained in the discrete test space, i.e. $V_{h, p} \subset V_{h, r}$, our practical DPG method delivers the optimal solution. Therefore, the stability at the discrete level is inherited from the continuous problem $\gamma_{h} \geq \gamma$ by construction [10]. Finally, we know from [20] that the discrete inf-sup condition implies the existence of a Fortin operator $\Pi$ that is idempotent with constant $C_{\Pi} \geq \frac{M}{\gamma_{h}}$. In Theorem 1 , we constructed such idempotent Fortin operator explicitly and, in addition, we proved that it is the orthogonal projection of $V$ into $V_{h, r}$.

Therefore, in this article we present a practical DPG method that delivers the optimal solution and an approximate error representation function $\psi_{h}$ that satisfies (50). In the numerical results in the next section, we employ the element contributions of $\left\|\psi_{h}\right\|_{V}$ as error indicators to guide the tadaptivity in time. For that, we employ the classical Dörfler marking strategy [17] with parameter $\theta \in[0,1]$. Note that, as we have a time-marching scheme, we only need to re-compute the values of the solution in the elements to the right of the first marked element in each iteration.

\section{Numerical results}

In this section, we present numerical results of adaptivity in time for both parabolic and hyperbolic problems. For the space discretization, we employ a Bubnov-Galerkin method with piecewise linear functions. As in [33,34], we employ the expression of the optimal test functions in terms of the $\varphi$-functions from exponential integrators in both the time marching (11) scheme and the error representation function (33). We employ the EXPINT [2] package in MATLAB for the evaluation of the corresponding $\varphi$-functions. 


\subsection{Approximation of the Riesz representation}

Here, we show an example of how close is the solution of (32) to (13) for a given righthand-side. First, we consider a single element and we define the following functional

$$
\mathcal{R}(v)=\int_{0}^{1} f(t) v(t) d t
$$

with $f(t)=t^{p}$ and $p \geq 0$. and we solve the following Riesz representation problem

$$
\left\{\begin{array}{l}
\text { Find } \psi \in V \text { such that } \\
(\psi, \delta v)_{V}=\mathcal{R}(\delta v), \quad \forall \delta v \in V
\end{array}\right.
$$

and also its discrete version

$$
\left\{\begin{array}{l}
\text { Find } \psi_{h} \in V_{h, r} \text { such that } \\
\left(\psi_{h}, \delta v_{h}\right)_{V}=\mathcal{R}\left(\delta v_{h}\right), \quad \forall \delta v_{h} \in V_{h, r} .
\end{array}\right.
$$

We know the analytical solution $\psi$ from Proposition 1 and we study its approximation $\psi_{h}$ for different values of $r, p$ and $\lambda$.

Figure 2 shows the solution of (54) and (55) for $\lambda=1$ and different values of $p$ and $r$. Table 1 displays the relative $L^{2}$-error for $\lambda=1$ and different values of $p$ and $r$. Figure 3 presents the solutions for $p=0, r=1$ and different values of $\lambda$. Table 2 displays the relative $L^{2}$-error for $\lambda=1$ and different values of $p$ and $r$. We observe that as we increase $r$, the practical error representation function $\psi_{h}$ better approximates the analytical one $\psi$. In practice, we select $r=p+1$.

\begin{tabular}{|c|c|c|c|c|}
\hline & $r=0$ & $r=1$ & $r=2$ & $r=3$ \\
\hline$p=0$ & $7.09 \cdot 10^{-2}$ & $\mathbf{5 . 9 9} \cdot \mathbf{1 0}^{-\mathbf{3}}$ & $3.54 \cdot 10^{-4}$ & $1.71 \cdot 10^{-5}$ \\
$p=1$ & $8.27 \cdot 10^{-2}$ & $1.12 \cdot 10^{-2}$ & $\mathbf{6 . 6 3} \cdot \mathbf{1 0}^{-4}$ & $3.19 \cdot 10^{-5}$ \\
$p=2$ & $7.09 \cdot 10^{-2}$ & $2.04 \cdot 10^{-2}$ & $1.93 \cdot 10^{-3}$ & $\mathbf{9 . 3 4} \cdot \mathbf{1 0}^{-\mathbf{5}}$ \\
\hline
\end{tabular}

Table 1: Relative $L^{2}$-error $\left\|\psi-\psi_{h}\right\| /\|\psi\|$ (Figure 2) for $\lambda=1$ and different values of $p$ and $r$.

\begin{tabular}{|c|c|c|c|c|c|}
\hline$\lambda=1$ & $\lambda=0.1$ & $\lambda=5$ & $\lambda=-1$ & $\lambda=-0.1$ & $\lambda=-5$ \\
\hline $5.99 \cdot 10^{-3}$ & $4.48 \cdot 10^{-4}$ & $3.02 \cdot 10^{-2}$ & $2.42 \cdot 10^{-3}$ & $4.09 \cdot 10^{-4}$ & $1.45 \cdot 10^{-2}$ \\
\hline
\end{tabular}

Table 2: Relative $L^{2}$-error $\left\|\psi-\psi_{h}\right\| /\|\psi\|$ (Figure 3) for $p=0, r=1$ and different values of $\lambda$. 

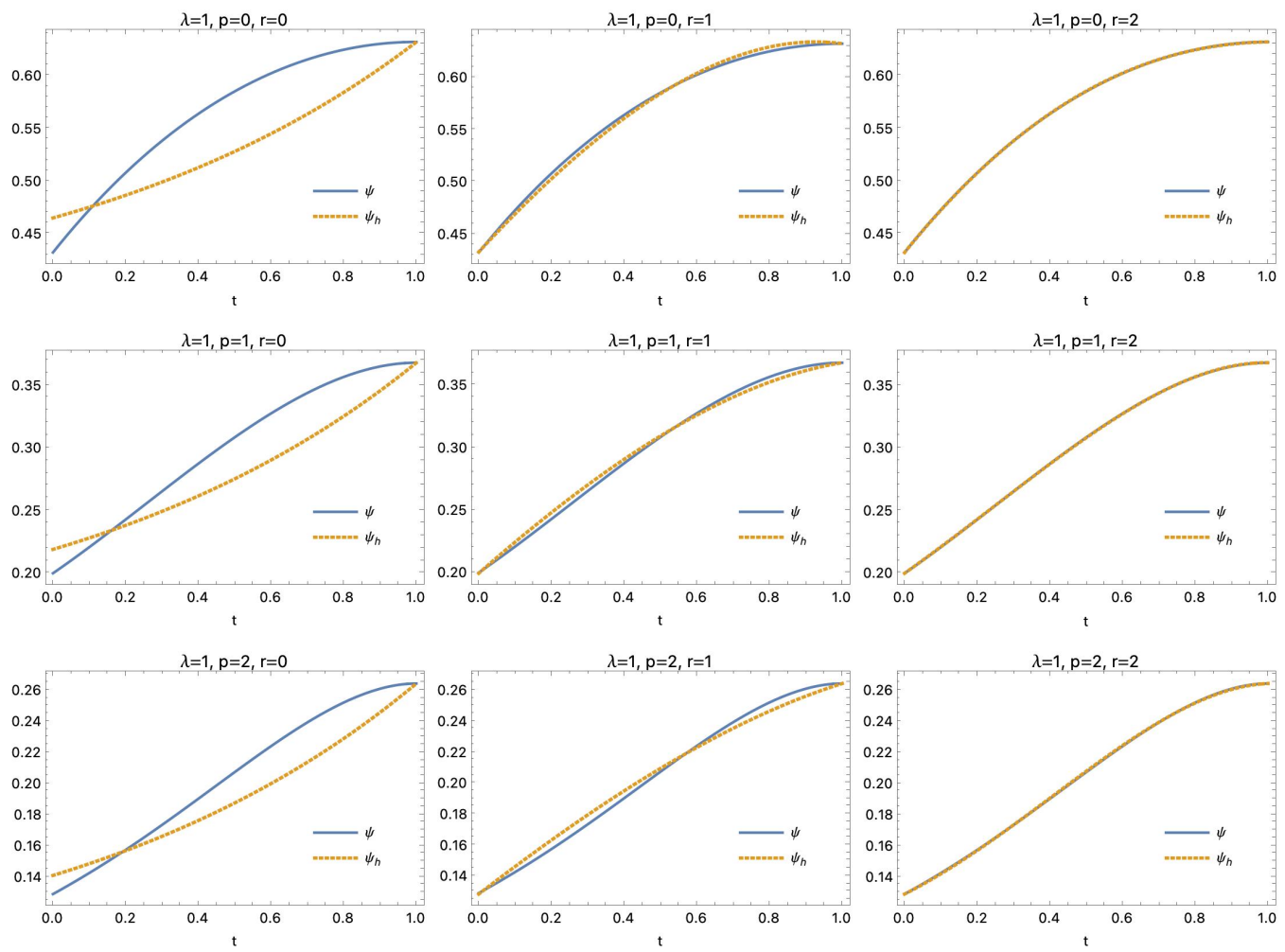

Figure 2: Functions $\psi$ and $\psi_{h}$ for $\lambda=1$ and different values of $p$ and $r$.
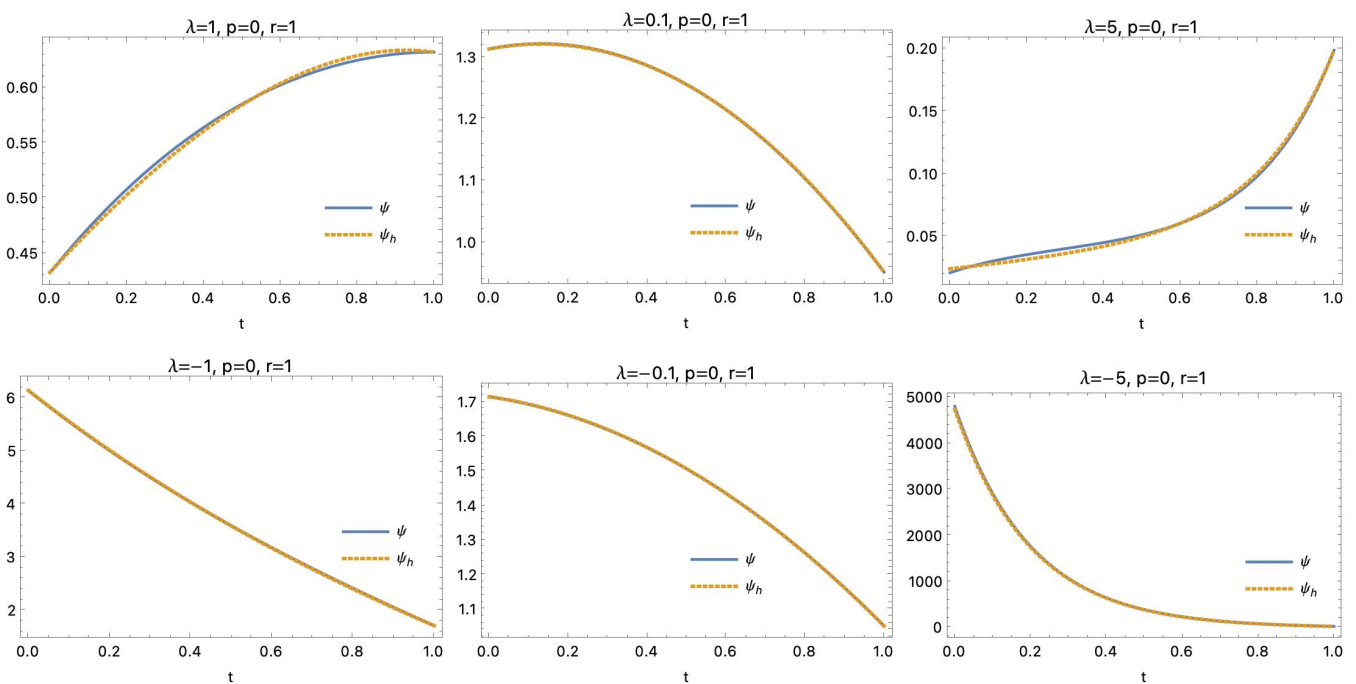

Figure 3: Functions $\psi$ and $\psi_{h}$ for $p=0, r=1$ and different values of $\lambda$. 


\subsection{Parabolic problem: single $O D E$}

We consider a similar example as in [34]. In (1), we set $f(t)=\frac{M}{e^{M}-1}, M=30$, $\lambda=-M$ and $I=(0,1]$. The exact solution to this problem is

$$
u(t)=\frac{e^{M(t-1)}-e^{-M}}{1-e^{-M}} .
$$

Figure 4 shows the convergence of the exact error for $p=0,1,2$ for uniform refinements and for adaptive refinements employing the Dörfler strategy with $\theta=0.5$ and enriched test space with $r=p+1$. We conclude that to achieve a desirable error, the adaptive strategy needs about an order of magnitude less degrees of freedom than when employing uniform refinements. Figure 5 presents the adapted solutions for $p=0,1,2$ for a fixed tolerance error and the corresponding local discrete error contribution function $-\left(\psi_{h}^{k}(t)\right)^{\prime}+\lambda \psi_{h}^{k}(t)$. Note that from (9) and Corollary 3, these functions are piecewise polynomials of order $r$ at each element. Finally, Figure 6 shows the convergence of the exact error for $p=0,1,2$ with uniform refinements and the convergence of the discrete error $\left\|\psi_{h}\right\|_{V}$ for $r=p+1$. We conclude that $\left\|\psi_{h}\right\|_{V}$ is an efficient and reliable error control to perform adaptivity.

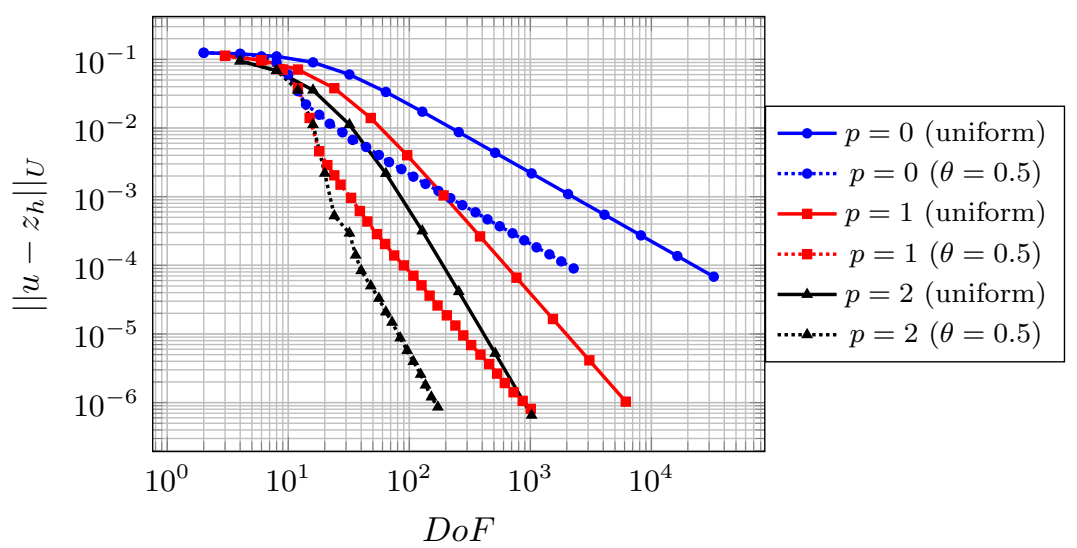

Figure 4: Convergence of the exact error for $p=0, p=1$ and $p=2$ performing uniform refinements and the Dörfler adaptive strategy with $\theta=0.5$. 

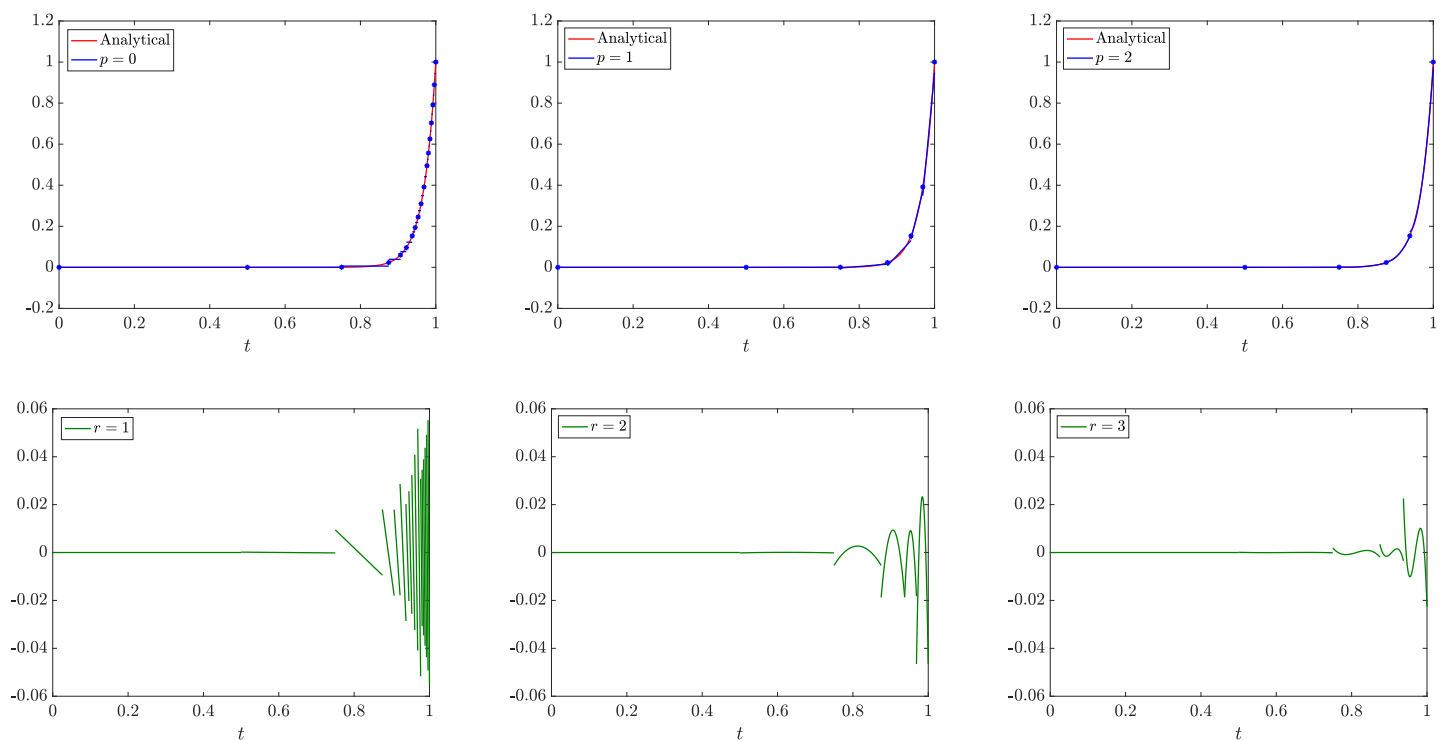

Figure 5: Adapted solution with $p=0, p=1$ and $p=2$ for a fixed tolerance error (top row) and the corresponding local error contributions for $r=p+1$ (bottom row).

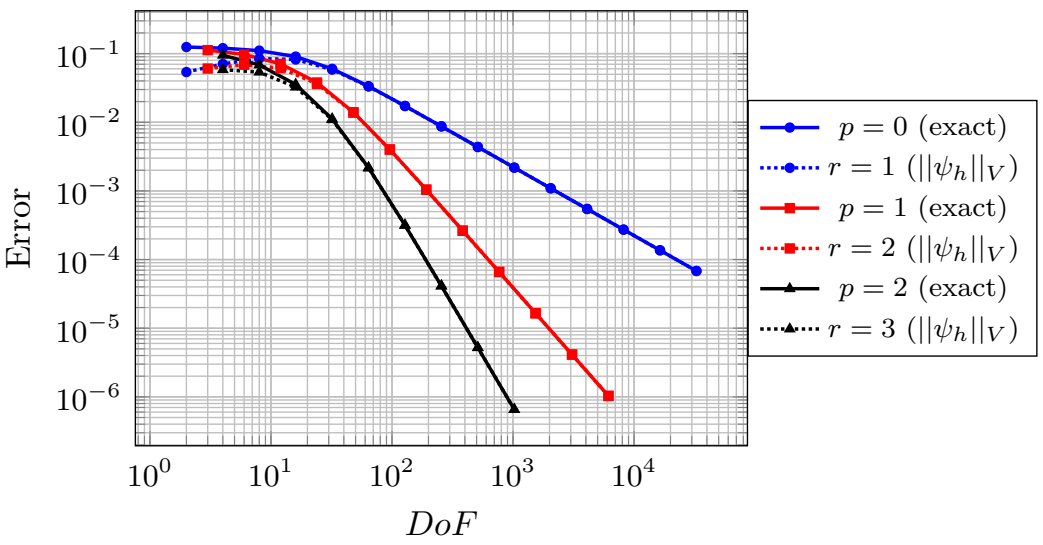

Figure 6: Convergence of the exact error for $p=0, p=1$ and $p=2$ and the error estimator $\left\|\psi_{h}\right\|_{V}$ for $r=p+1$ when performing uniform refinements. 
6.3. Parabolic problem: single ODE with a strong gradient

We consider in (1) the data corresponding with the following exact solution

$$
u(t)=\left(1+e^{-M\left(t-\frac{1}{2}\right)}\right)^{-1},
$$

with $\lambda=M=10^{3}$, which has strong gradientes nearby $t=\frac{1}{2}$. Figure 7 compares the convergence of the exact error for uniform vs adaptive refinements with $\theta=0.5$. Figure 8 displays the adaptive solution and error contributions for $p=1,2$ and $r=p+1$ for a fixed tolerance. We conclude that the error representation captures the strong gradient of the solution and performs refinements around $t=\frac{1}{2}$.

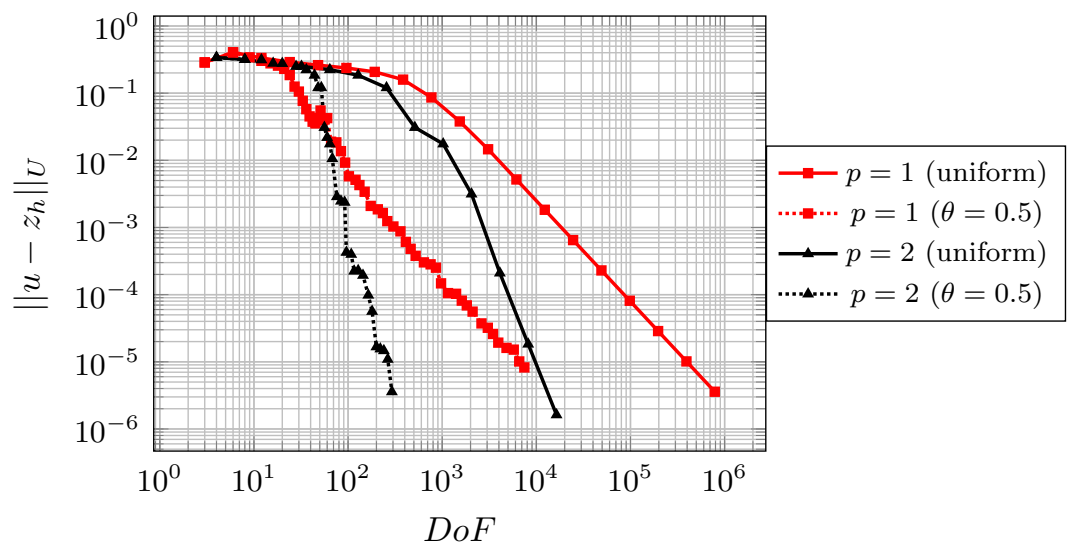

Figure 7: Convergence of the exact error for $p=1,2$ performing uniform refinements and the Dörfler adaptive strategy with $\theta=0.5$. 

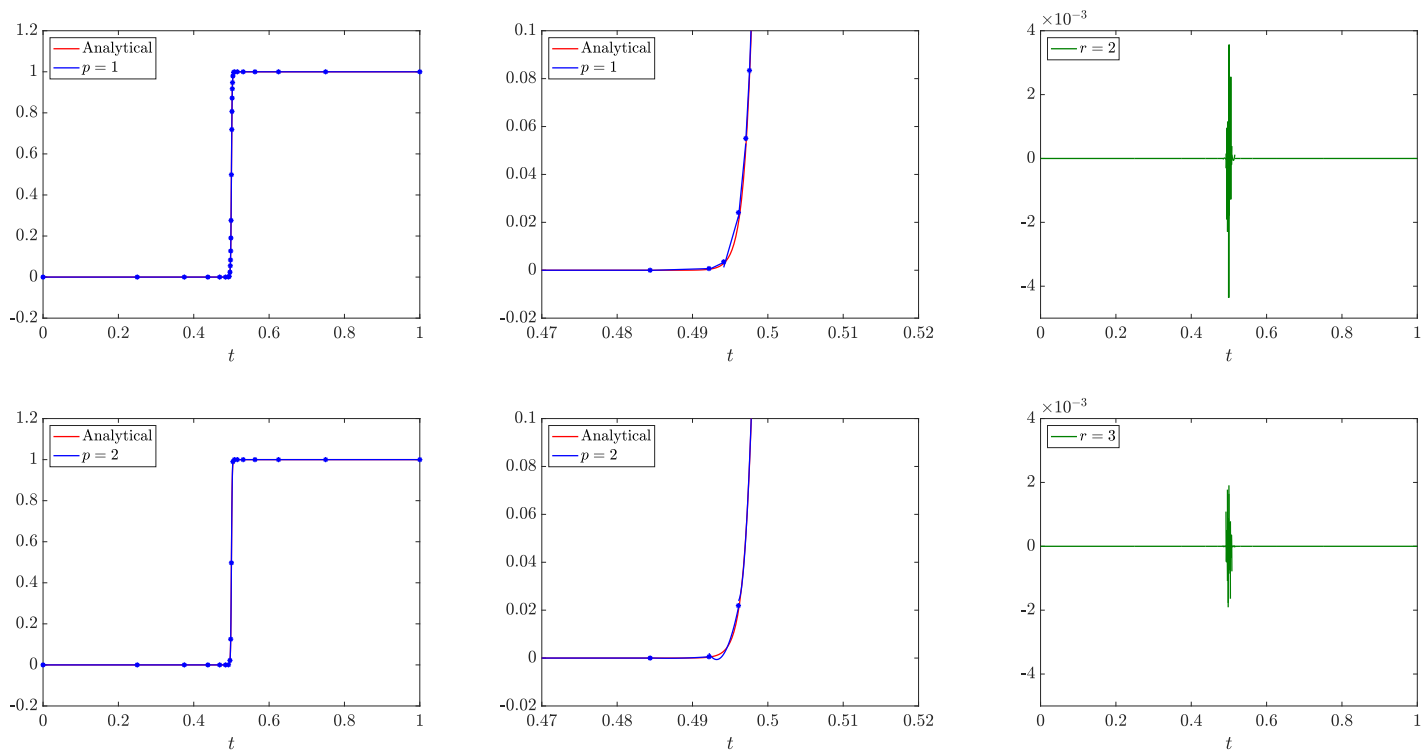

Figure 8: Adapted solution with $p=1,2$ for a fixed tolerance error and local error contributions for $r=p+1$.

\subsection{Parabolic problem: $1 D+$ time}

We consider the following 1D+time parabolic problem that we introduced in [34]

$$
\left\{\begin{array}{lr}
\frac{\partial u}{\partial t}-\alpha^{2} \frac{\partial^{2} u}{\partial x^{2}}=f(x, t), & \forall(x, t) \in \Omega \times I, \\
u(x, t)=0, & \forall(x, t) \in \partial \Omega \times I, \\
u(x, 0)=u_{0}(x), & \forall x \in \Omega .
\end{array}\right.
$$

We set $\Omega=(0,1), I=(0,0.5]$ and the data of the problem corresponding to the exact solution

$$
u(x, t)=e^{-2 \pi^{2} t} \sin (\pi x) .
$$

Figure 9 shows the adapted solutions and cross sections at $x=0.5$ for $p=0,1,2$ and 600 elements in space and $\theta=0.5$. Figure 10 presents the corresponding error contribution functions and their cross sections at $x=0.5$. In this case, the errors are space-time tensor products with polynomials of order $r=p+1$ in time. Figure 11 compares the relative error of the solutions when we perform uniform refinements and adaptivity with 600 elements in space and $\theta=0.5$. We conclude that the adaptive strategy is more efficient. Finally, Figure 12 shows the convergence of the relative errors when we employ 50 elements in space. We observe that the adaptive strategy in time stops converging when the error in space becomes dominant, as expected. 

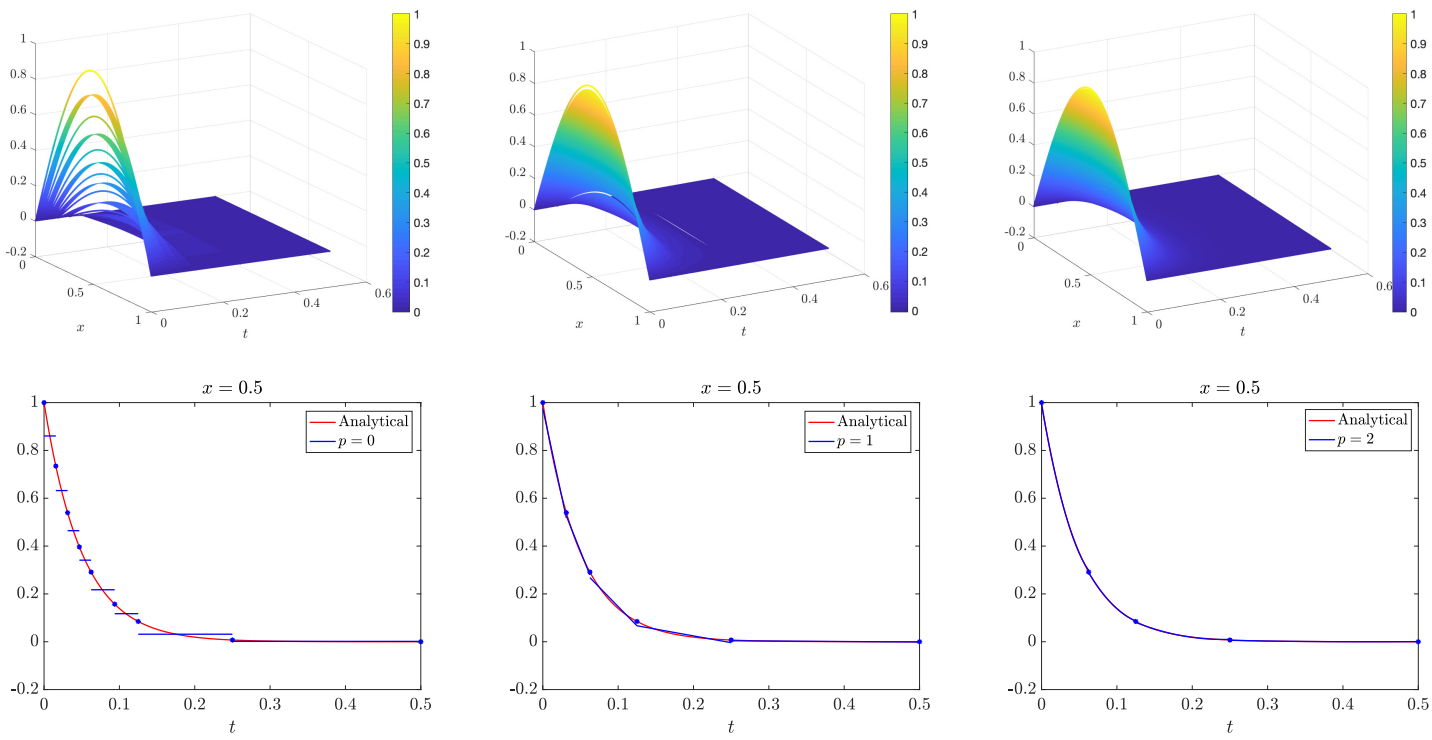

Figure 9: Adapted solution with $p=0, p=1$ and $p=2$ for a fixed tolerance error (top row) and the corresponding cross sections at $x=0.5$ (bottom row).
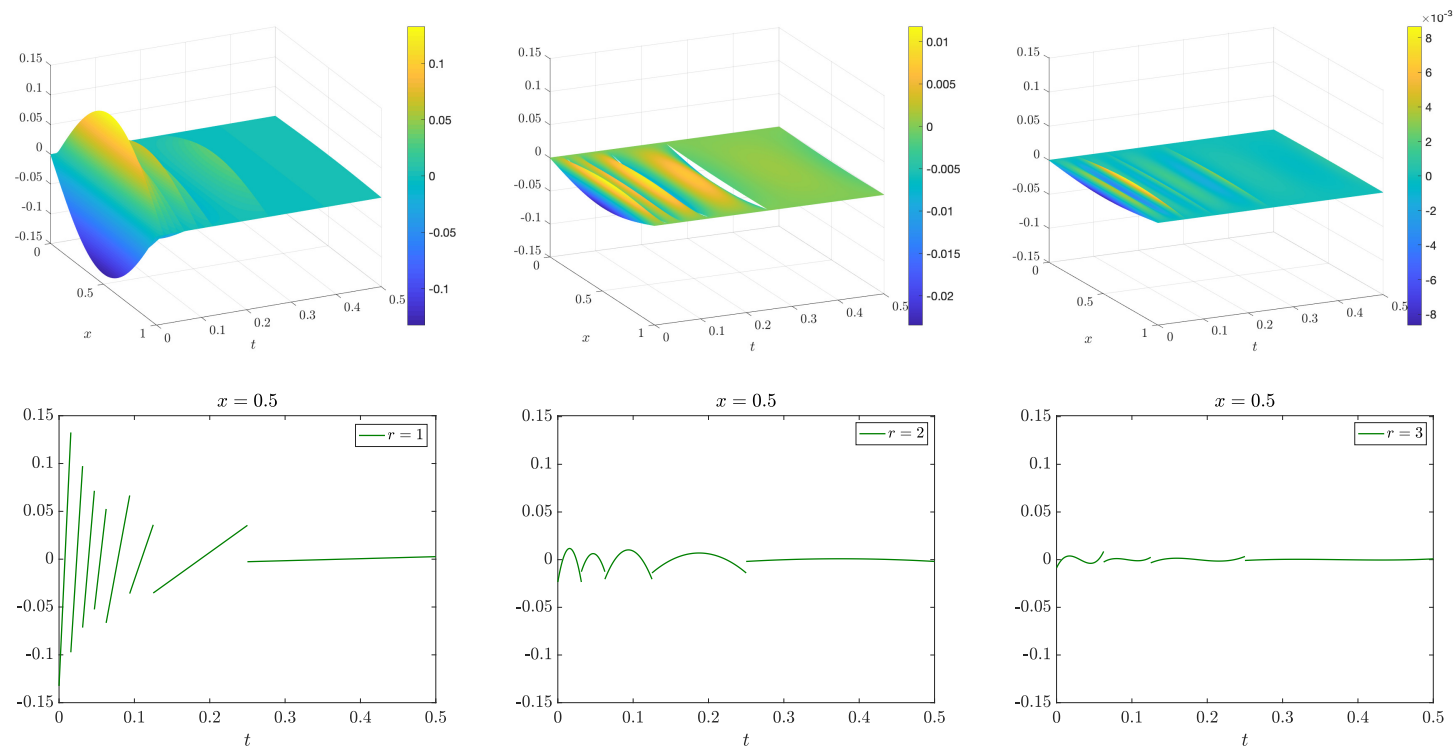

Figure 10: Error representation functions with $p=0, p=1$ and $p=2$ for a fixed tolerance error (top row) and the corresponding cross sections at $x=0.5$ (bottom row). 


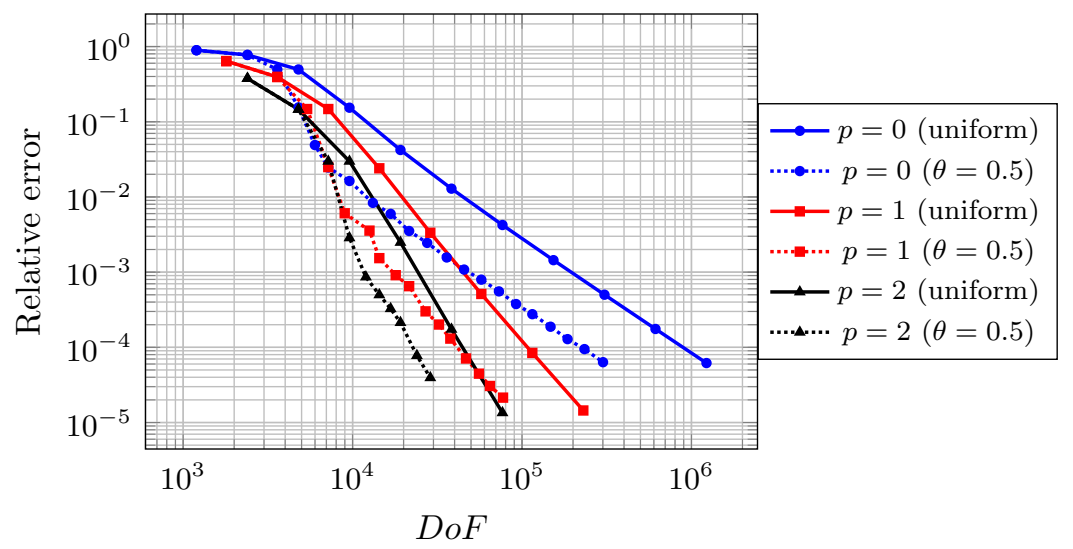

Figure 11: Relative error for $p=0, p=1$ and $p=2$ when performing uniform refinements and the Dörfler adaptive strategy with $\theta=0.5$. Numer of elements in space: 600 .

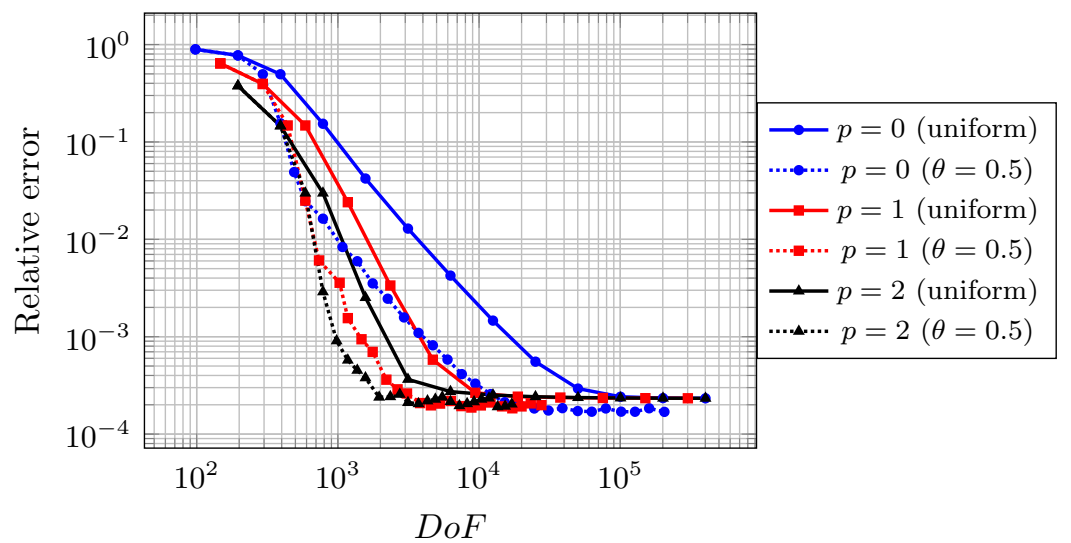

Figure 12: Relative error for $p=0, p=1$ and $p=2$ when performing uniform refinements and the Dörfler adaptive strategy with $\theta=0.5$. Number of elements in space: 50 . 


\subsection{Hyperbolic problem}

We consider the model hyperbolic problem presented in [33]

$$
\left\{\begin{aligned}
U^{\prime}(t)+A U(t) & =F(t), \quad \text { in } I, \\
U(0) & =U_{0},
\end{aligned}\right.
$$

where $v=u^{\prime}$ and

$$
U(t)=\left[\begin{array}{l}
u(t) \\
v(t)
\end{array}\right], A=\left[\begin{array}{cc}
0 & -1 \\
\alpha^{2} & 0
\end{array}\right], F(t)=\left[\begin{array}{c}
0 \\
f(t)
\end{array}\right], U_{0}=\left[\begin{array}{l}
u_{0} \\
v_{0}
\end{array}\right]
$$

In this example, we set the data corresponding to the following exact solution

$$
U(t)=\left[\begin{array}{c}
e^{\beta t} \sin (\gamma t) \\
\beta e^{\beta t} \sin (\gamma t)+\gamma e^{\beta t} \cos (\gamma t)
\end{array}\right]
$$

in $I=(0,1]$ with $\beta=-4 \pi$ and $\gamma=18 \pi$.

Figure 13 displays the convergence of the exact error for $p=0,1,2$ for uniform refinements and for adaptive refinements fixing $\theta=0.5$ and $r=p+1$. Figures 14 and 15 present the adapted solutions for $p=0,1,2$ and the corresponding local error contribution functions for $u(t)$ and $v(t)$, respectively. In this case, as explained in [34], the source is time dependent and $p=0$ is insufficient to obtain a good approximation since we would require a very fine mesh. We observe here that for $p=2$, the error of integrating the source is of lowest order and we obtain a good adapted solution after a few iterations.

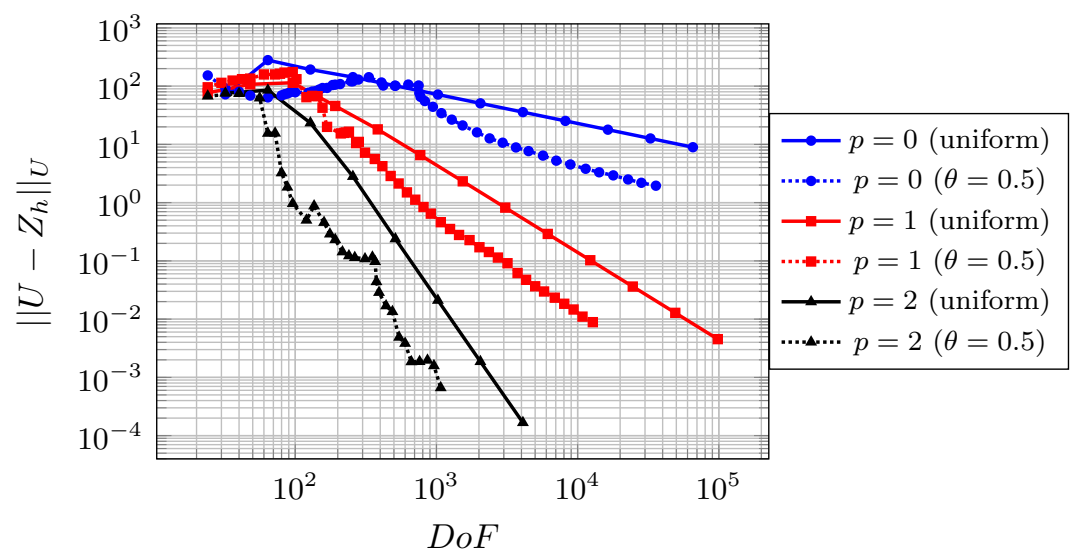

Figure 13: Convergence of the exact error for $p=0, p=1$ and $p=2$ performing uniform refinements and the Dörfler adaptive strategy with $\theta=0.5$. 

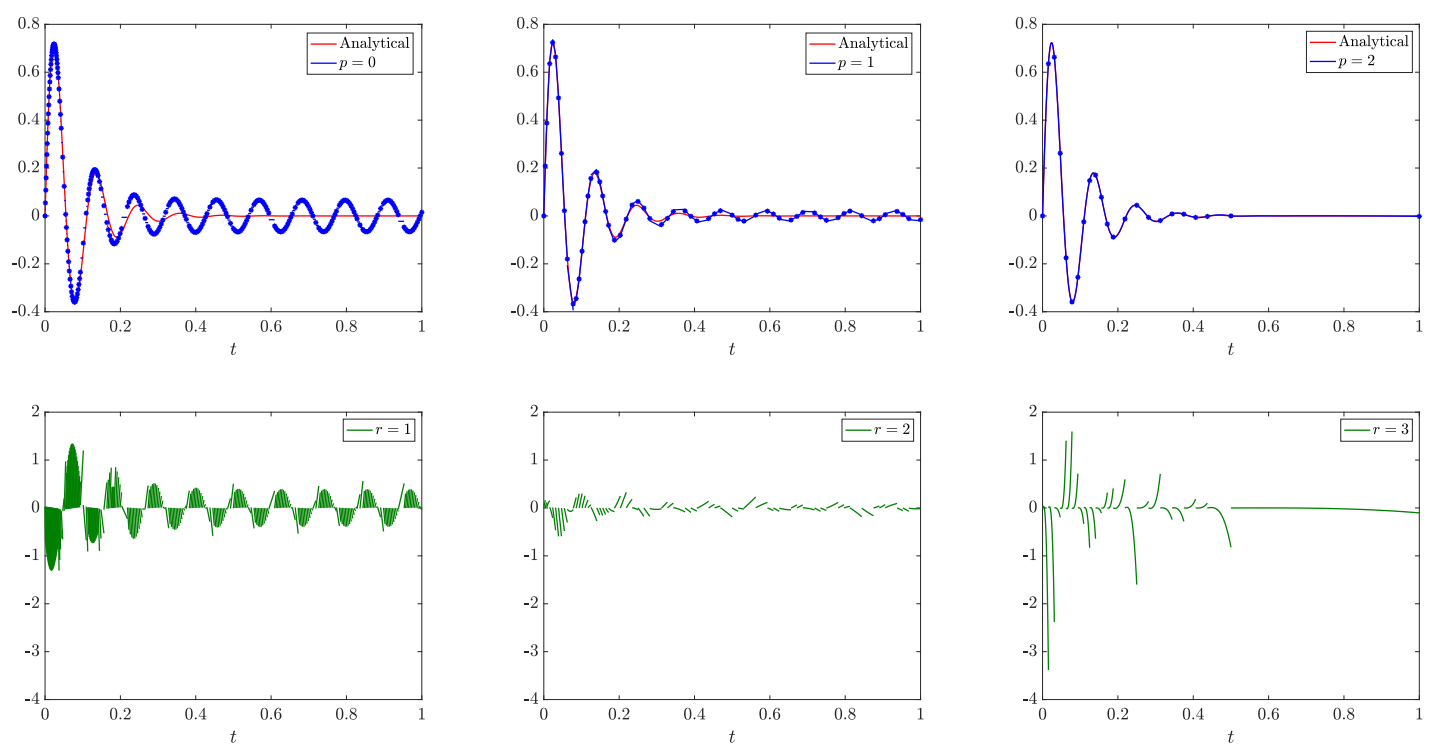

Figure 14: Adapted solution of $u(t)$ with $p=0, p=1$, and $p=2$ for a fixed tolerance error (top row) and the corresponding local error contributions for $r=p+1$ (bottom row).
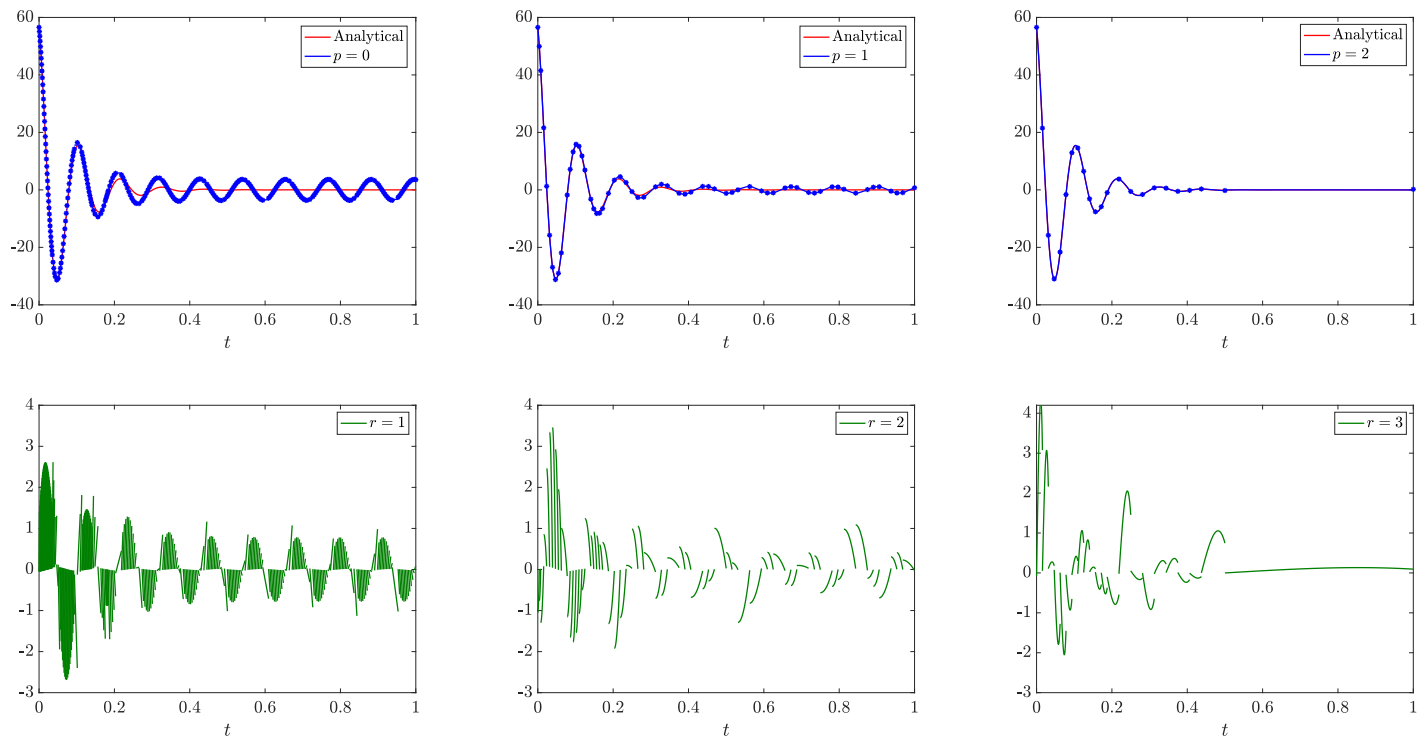

Figure 15: Adapted solution of $v(t)$ with $p=0, p=1$, and $p=2$ for a fixed tolerance error (top row) and the corresponding local error contributions for $r=p+1$ (bottom row). 


\section{Conclusions and future work}

In this article, we study an error representation function to perform adaptivity in time in the DPG time-marching scheme we recently introduced in $[33,34]$. We apply the DPG method in the time variable only so we can compute analytically the error representation function by inverting the Riesz operator of the residual. However, in order to obtain computable error estimators, we approximate the analytical error by enriching the test space. The enriched test space we propose contains the analytical optimal test functions so our method still delivers the optimal DPG solution. We compute both the solution and the error contributions in a time marching-scheme that has a few more equations than the ones presented in $[33,34]$. We prove via analysis confirmed with numerical evidence that our proposed approximation error is reliable and efficient to perform adaptivity.

Possible extensions of this work include: (a) to combine the adaptivity in time together with adaptivity in space for the Bubnov-Galerkin method; (b) to combine the adaptive DPG-based time-marching scheme together with DPG in space; (c) to design goal-oriented adaptive strategies; (d) to extend the method to non-linear problems.

\section{Acknowledgements}

Judit Muñoz-Matute and David Pardo have received funding from the European Union's Horizon 2020 research and innovation programme under the Marie Sklodowska-Curie grant agreement No 777778 (MATHROCKS), the Projects of the Spanish Ministry of Science and Innovation with references PID2019-108111RB-I00 (FEDER/AEI) and PDC2021-121093I00, the BCAM "Severo Ochoa" accreditation of excellence (SEV-2017-0718), and the Basque Government through the BERC 2018-2021 program, and the Consolidated Research Group MATHMODE (IT1294-19) given by the Department of Education.

Judit Muñoz-Matute has also received founding from the Basque Government through the postdoctoral program for the improvement of doctor research staff (POS_2019_1_0001) and the European Union's Horizon 2020 research and innovation programme under the Marie Sklodowska-Curie individual fellowship grant agreement No 101017984 (GEODPG).

David Pardo has also received funding from the European POCTEFA 2014-2020 Project PIXIL (EFA362/19) by the European Regional Development Fund (ERDF) through the Interreg V-A Spain-France-Andorra programme and the three Elkartek projects 3KIA (KK2020/00049), EXPERTIA (KK-2021/00048), and SIGZE (KK-2021/00095).

Leszek Demkowicz was partially supported with NSF grant No. 1819101.

\section{References}

[1] A. V. Astaneh, F. Fuentes, J. Mora, and L. Demkowicz. High-order polygonal discontinuous Petrov-Galerkin (PolyDPG) methods using ultraweak formulations. Computer Methods in Applied Mechanics and Engineering, 332:686-711, 2018. 
[2] H. Berland, B. Skaflestad, and W. M. Wright. EXPINT-A MATLAB package for exponential integrators. ACM Transactions on Mathematical Software (TOMS), 33(1):4es, 2007.

[3] C. Carstensen, L. Demkowicz, and J. Gopalakrishnan. A posteriori error control for DPG methods. SIAM Journal on Numerical Analysis, 52(3):1335-1353, 2014.

[4] C. Carstensen, L. Demkowicz, and J. Gopalakrishnan. Breaking spaces and forms for the DPG method and applications including Maxwell equations. Computers 83 Mathematics with Applications, 72(3):494-522, 2016.

[5] J. Chan, N. Heuer, T. Bui-Thanh, and L. Demkowicz. A robust DPG method for convection-dominated diffusion problems II: Adjoint boundary conditions and meshdependent test norms. Computers $\& 3$ Mathematics with Applications, 67(4):771-795, 2014.

[6] L. Demkowicz, T. Führer, N. Heuer, and X. Tian. The double adaptivity paradigm: (How to circumvent the discrete inf-sup conditions of Babuška and Brezzi). Computers \& Mathematics with Applications, 95:41-66, 2021.

[7] L. Demkowicz and J. Gopalakrishnan. A class of discontinuous Petrov-Galerkin methods. Part I: The transport equation. Computer Methods in Applied Mechanics and Engineering, 199(23-24):1558-1572, 2010.

[8] L. Demkowicz and J. Gopalakrishnan. Analysis of the DPG method for the Poisson equation. SIAM Journal on Numerical Analysis, 49(5):1788-1809, 2011.

[9] L. Demkowicz and J. Gopalakrishnan. A class of discontinuous Petrov-Galerkin methods. Part II: Optimal test functions. Numerical Methods for Partial Differential Equations, 27(1):70-105, 2011.

[10] L. Demkowicz and J. Gopalakrishnan. An overview of the discontinuous PetrovGalerkin method. In Recent developments in discontinuous Galerkin finite element methods for partial differential equations, pages 149-180. Springer, 2014.

[11] L. Demkowicz and J. Gopalakrishnan. Discontinuous Petrov-Galerkin (DPG) method. Encyclopedia of Computational Mechanics Second Edition, pages 1-15, 2017.

[12] L. Demkowicz, J. Gopalakrishnan, and B. Keith. The DPG-star method. Computers E Mathematics with Applications, 79(11):3092-3116, 2020.

[13] L. Demkowicz, J. Gopalakrishnan, I. Muga, and J. Zitelli. Wavenumber explicit analysis of a DPG method for the multidimensional Helmholtz equation. Computer Methods in Applied Mechanics and Engineering, 213:126-138, 2012.

[14] L. Demkowicz, J. Gopalakrishnan, S. Nagaraj, and P. Sepúlveda. A spacetime DPG method for the Schrödinger equation. SIAM Journal on Numerical Analysis, 55(4):1740-1759, 2017. 
[15] L. Demkowicz, J. Gopalakrishnan, and A. H. Niemi. A class of discontinuous PetrovGalerkin methods. Part III: Adaptivity. Applied numerical mathematics, 62(4):396427, 2012.

[16] L. Demkowicz and N. Heuer. Robust DPG method for convection-dominated diffusion problems. SIAM Journal on Numerical Analysis, 51(5):2514-2537, 2013.

[17] W. Dörfler. A convergent adaptive algorithm for Poisson's equation. SIAM Journal on Numerical Analysis, 33(3):1106-1124, 1996.

[18] T. Ellis, J. Chan, and L. Demkowicz. Robust DPG methods for transient convectiondiffusion. In Building bridges: connections and challenges in modern approaches to numerical partial differential equations, pages 179-203. Springer, 2016.

[19] T. Ellis, L. Demkowicz, J. Chan, and R. Moser. Space-time DPG: Designing a method for massively parallel CFD. ICES report. The Institute for Computational Engineering and Sciences, The University of Texas at Austin, pages 14-32, 2014.

[20] A. Ern and J.-L. Guermond. A converse to fortin's lemma in banach spaces. Comptes Rendus Mathematique, 354(11):1092-1095, 2016.

[21] M. Fortin. An analysis of the convergence of mixed finite element methods. RAIRO. Analyse numérique, 11(4):341-354, 1977.

[22] T. Führer, N. Heuer, and J. S. Gupta. A time-stepping DPG scheme for the heat equation. Computational Methods in Applied Mathematics, 17(2):237-252, 2017.

[23] T. Führer, N. Heuer, and M. Karkulik. Analysis of backward Euler primal DPG methods. arXiv preprint arXiv:2103.12181, 2021.

[24] T. Führer and M. Karkulik. Space-time least-squares finite elements for parabolic equations. Computers \& Mathematics with Applications, 92:27-36, 2021.

[25] J. Gopalakrishnan and W. Qiu. An analysis of the practical DPG method. Mathematics of Computation, 83(286):537-552, 2014.

[26] J. Gopalakrishnan and P. Sepúlveda. A space-time DPG method for the wave equation in multiple dimensions. Space-Time Methods. Applications to Partial Differential Equations, pages 129-154, 2017.

[27] S. Henneking and L. Demkowicz. A numerical study of the pollution error and DPG adaptivity for long waveguide simulations. Computers \&s Mathematics with Applications, 95:85-100, 2021.

[28] N. J. Higham and E. Hopkins. A catalogue of software for matrix functions. version 3.0. 2020. 
[29] M. Hochbruck and A. Ostermann. Exponential integrators. Acta Numerica, 19:209$286,2010$.

[30] M. Hochbruck and A. Ostermann. Exponential multistep methods of Adams-type. BIT Numerical Mathematics, 51(4):889-908, 2011.

[31] M. Hochbruck, A. Ostermann, and J. Schweitzer. Exponential Rosenbrock-type methods. SIAM Journal on Numerical Analysis, 47(1):786-803, 2009.

[32] B. Keith, A. V. Astaneh, and L. F. Demkowicz. Goal-oriented adaptive mesh refinement for discontinuous Petrov-Galerkin methods. SIAM Journal on Numerical Analysis, 57(4):1649-1676, 2019.

[33] J. Muñoz-Matute, D. Pardo, and L. Demkowicz. A DPG-based time-marching scheme for linear hyperbolic problems. Computer Methods in Applied Mechanics and Engineering, 373:113539, 2021.

[34] J. Muñoz-Matute, D. Pardo, and L. Demkowicz. Equivalence between the DPG method and the exponential integrators for linear parabolic problems. Journal of Computational Physics, 429:110016, 2021.

[35] S. Nagaraj, S. Petrides, and L. F. Demkowicz. Construction of DPG Fortin operators for second order problems. Computers \& Mathematics with Applications, 74(8):19641980, 2017.

[36] S. Petrides and L. F. Demkowicz. An adaptive DPG method for high frequency timeharmonic wave propagation problems. Computers $\&$ Mathematics with Applications, 74(8):1999-2017, 2017.

[37] N. V. Roberts and S. Henneking. Time-stepping DPG formulations for the heat equation. Computers $\& 3$ Mathematics with Applications, 95:242-255, 2021.

[38] S. Rojas, D. Pardo, P. Behnoudfar, and V. M. Calo. Goal-oriented adaptivity for a conforming residual minimization method in a dual discontinuous Galerkin norm. Computer Methods in Applied Mechanics and Engineering, 377:113686, 2021.

[39] E. Valseth and C. Dawson. An unconditionally stable space-time FE method for the Korteweg-de Vries equation. Computer Methods in Applied Mechanics and Engineering, 371:113297, 2020.

[40] E. Valseth, A. Romkes, and A. R. Kaul. A stable FE method for the space-time solution of the Cahn-Hilliard equation. Journal of Computational Physics, 441:110426, 2021. 